\title{
Activating transcription factor 4 regulates osteoclast differentiation in mice
}

\author{
Huiling Cao,, ${ }^{1,2}$ Shibing Yu, ${ }^{1}$ Zhi Yao, ${ }^{2}$ Deborah L. Galson, ${ }^{1}$ Yu Jiang, ${ }^{3}$ Xiaoyan Zhang, ${ }^{1,4}$ Jie Fan, ${ }^{5}$ \\ Binfeng Lu, 6 Youfei Guan, ${ }^{4}$ Min Luo,, ${ }^{1}$ Yumei Lai, ${ }^{3}$ Yibei Zhu, ${ }^{6}$ Noriyoshi Kurihara, ${ }^{1}$ \\ Kenneth Patrene, ${ }^{1}$ G. David Roodman, ${ }^{1}$ and Guozhi Xiao ${ }^{1,7}$
}

\begin{abstract}
${ }^{1}$ Department of Medicine, University of Pittsburgh, Pittsburgh, Pennsylvania, USA. ${ }^{2}$ Department of Immunology, Key Laboratory of Educational Ministry of China, Tianjin Medical University, Tianjin, China. ${ }^{3}$ Department of Pharmacology and Chemical Biology, University of Pittsburgh, Pittsburgh, Pennsylvania, USA. ${ }^{4}$ Department of Physiology and Pathophysiology, Peking University Health Science Center, Beijing, China. ${ }^{5}$ Department of Surgery and ${ }^{6}$ Department of Immunology, University of Pittsburgh, Pittsburgh, Pennsylvania, USA. ${ }^{7}$ College of Life Sciences, Nankai University, Tianjin, China.
\end{abstract}

\begin{abstract}
Activating transcription factor 4 (ATF4) is a critical transcription factor for osteoblast (OBL) function and bone formation; however, a direct role in osteoclasts (OCLs) has not been established. Here, we targeted expression of ATF4 to the OCL lineage using the Trap promoter or through deletion of Atf4 in mice. OCL differentiation was drastically decreased in Atf4-/- bone marrow monocyte (BMM) cultures and bones. Coculture of Atf4-/- BMMs with WT OBLs or a high concentration of RANKL failed to restore the OCL differentiation defect. Conversely, Trap-Atf4-tg mice displayed severe osteopenia with dramatically increased osteoclastogenesis and bone resorption. We further showed that ATF4 was an upstream activator of the critical transcription factor $N$ fatc 1 and was critical for RANKL activation of multiple MAPK pathways in OCL progenitors. Furthermore, ATF4 was crucial for M-CSF induction of RANK expression on BMMs, and lack of ATF4 caused a shift in OCL precursors to macrophages. Finally, ATF4 was largely modulated by M-CSF signaling and the PI3K/AKT pathways in BMMs. These results demonstrate that ATF4 plays a direct role in regulating OCL differentiation and suggest that it may be a therapeutic target for treating bone diseases associated with increased OCL activity.
\end{abstract}

\section{Introduction}

Skeletal integrity requires a delicate balance between bone-forming osteoblasts (OBLs) and bone-resorbing osteoclasts (OCLs). Abnormal osteoclastogenesis results in bone destruction, such as osteoporosis, metastatic osteolytic lesions, Paget disease of bone, and rheumatoid arthritis. In contrast, reduced osteoclastogenesis causes osteopetrosis, a disorder characterized by significantly increased skeletal mass and lack of a marrow space. Osteopetrosis is usually observed in animals or humans in which genes encoding cytokines, receptors, and signal transduction and transcription factors critical for OCL differentiation - such as RANKL; its receptor, RANK; the M-CSF receptor CSF1R (also known as c-Fms); TNF receptor-associated factor 6 (TRAF6); Src; PU.1, encoded by Spi1; p50/p52 NF-kb subunits; c-Fos; or v-ATPase $V_{0}$ subunit - are deleted or mutated (1-10). Defining the molecular mechanisms underlying osteoclastogenesis is essential to advance the understanding of the molecular basis for the pathogenesis of bone diseases with altered OCL activity. This knowledge will be important for the prevention and treatment of these diseases.

OCLs originate from cells in the monocyte/macrophage lineage (11). OCL formation and maturation are tightly regulated by OBL/stromal cell/hypertrophic chondrocyte-derived factors such as M-CSF, RANKL, and osteoprotegerin (OPG), a soluble decoy receptor that blocks RANKL binding to RANK and thereby inhibits OCL differentiation (12-15). M-CSF binds to its receptor, CSF1R, on early macrophage lineage cells and activates the Rank gene to generate OCL progenitors (16). The PI3K/AKT signaling pathways, which are strongly activated by M-CSF, play a critical role in activating OCL differentiation and bone resorption in nor-

Authorship note: Huiling Cao and Shibing Yu contributed equally to this work. Conflict of interest: The authors have declared that no conflict of interest exists. Citation for this article: J Clin Invest. 2010;120(8):2755-2766. doi:10.1172/JCI42106. mal and diseased states (17-24). However, little is known about the downstream molecular events that result from M-CSF-PI3K/ AKT signaling and their relationship to osteoclastogenesis.

RANKL, a member of the TNF superfamily, binds to RANK on OCL precursors and recruits TRAF6, resulting in the activation of multiple signaling pathways including IKK complexes (IKK $\alpha$, IKK $\beta$, IKK $\gamma$, and NIK-IKK $\alpha$ ) and MAPKs (Erk1/2, p38, and JNK) $(2,3,25)$, which leads to activation of critical transcription factors such as NF-KB and c-Fos. RANKL activation of NF- $\mathrm{KB}$ and c-Fos results in induction of initial expression of the key transcription factor NFATc1 (also designated as NFAT2 or NFATc), which is activated by the $\mathrm{Ca}^{2+} /$ calmodulin-regulated phosphatase calcineurin (3, 26-29). Eventually, calcium signaling occurs and activates the existing NFATc1, which triggers NFATc1 autoamplification (3, 30) required for further OCL differentiation. Inactivation of the Nfatc1 gene in vivo is embryonic lethal due to a heart valve defect $(31,32)$. However, the lack of rescue of osteopetrosis in OCL-deficient $\mathrm{c}$-Fos ${ }^{-1}$ mice by adoptive transfer of $\mathrm{Nfatc1}^{-/-}$(as opposed to $\mathrm{Nfatc1}^{+/-}$) hematopoietic stem cells provided evidence that NFATc1 is essential for osteoclastogenesis in vivo (33). Accordingly, transgenic overexpression of a constitutively active form of NFATc1 (caNFATc1) results in a skeletal phenotype of osteopenia associated with increased osteoclastogenesis and bone resorption (34). Importantly, overexpression of NFATc1 activates osteoclastogenesis in the absence of RANKL $(29,35,36)$. In addition, ectopic NFATc1 can restore osteoclastogenesis in both the p50/p52 double knockout (37) and the c-Fos knockout mouse (38). These results suggest that the major OCL differentiation signals converge on NFATc1. However, the molecular mechanisms that control the expression of this key factor are not completely understood.

Critical roles for ATF4 in OBLs and bone formation are well established. ATF4 favors bone formation by promoting OBL-specific gene expression, amino acid import and the synthesis of type I 
A

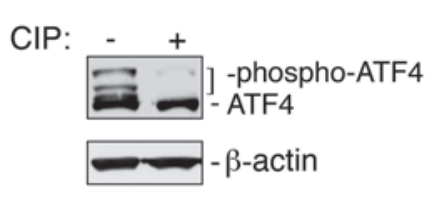

C
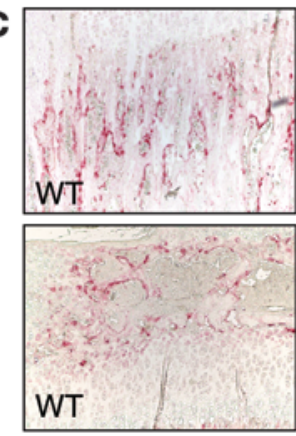

E

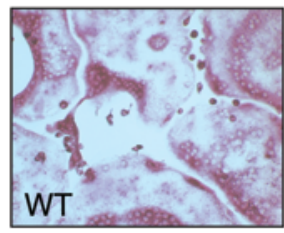

G

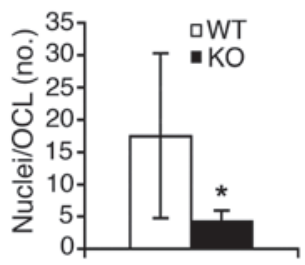

I

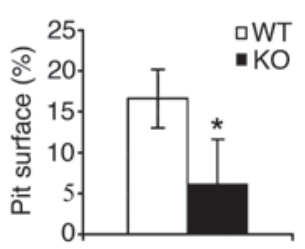

H

$\mathbf{J}$
B

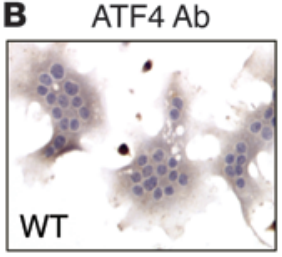

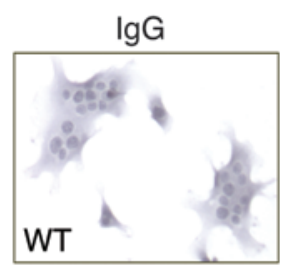

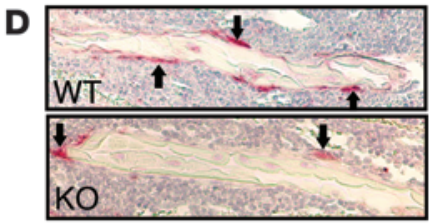

Ko
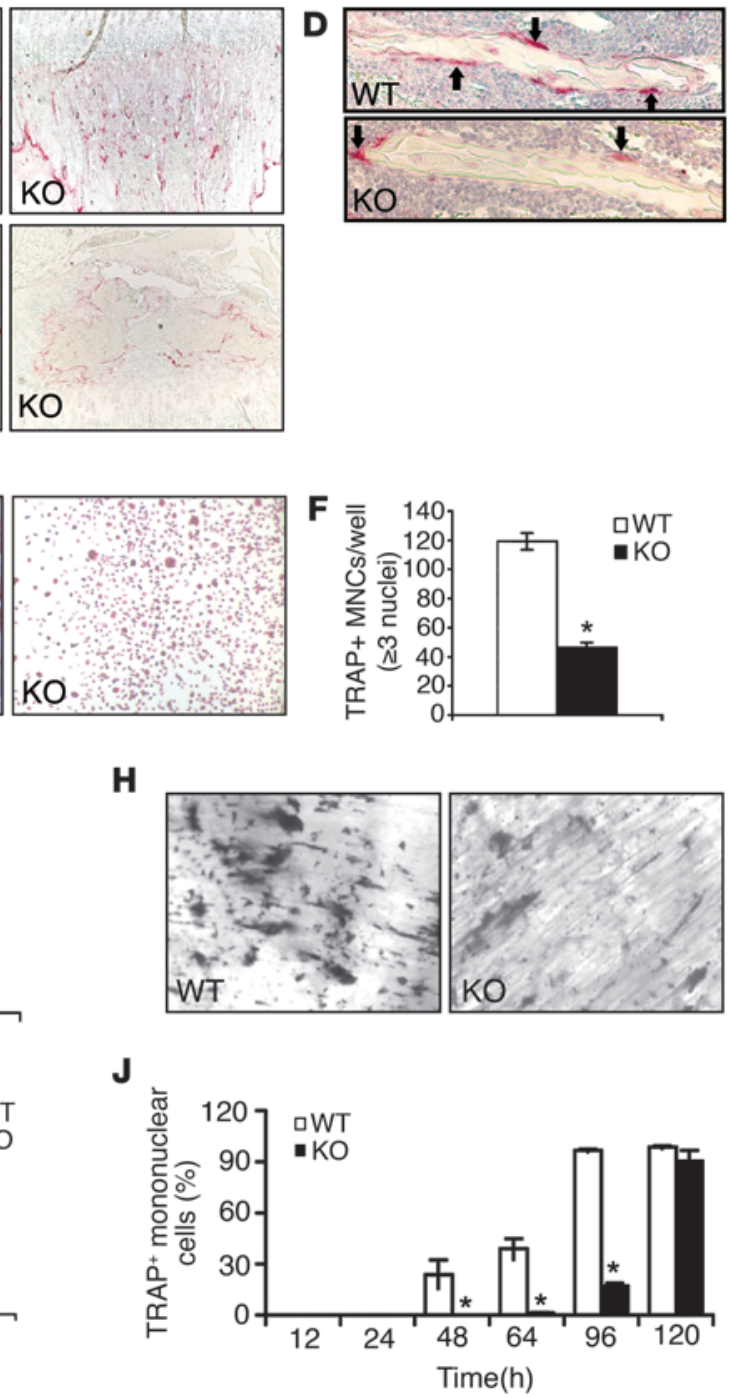

Figure 1

OCL differentiation is dramatically diminished in Atf4 $^{-1-}$ BMM cultures and bones. (A) Western blot. Whole cell extracts $(20 \mu \mathrm{g})$ from primary BMMs were incubated with or without 1 unit calf intestinal phosphatase (CIP) at room temperature for 30 minutes. (B) IHC. Differentiated BMMs were stained with an ATF4 antibody or control IgG. (C) Tibial sections were stained for TRAP activity for 30 minutes at $37^{\circ} \mathrm{C}$. TRAP activity in both metaphyseal (top) and epiphyseal (bottom) regions of tibias is shown. (D) TRAP+ OCLs (arrows) on trabecular surfaces of WT and $\mathrm{Atf}^{-/-}$tibiae. Oc.S/BS and Oc.Nb/BPm values for primary and secondary spongiosa are shown in Table 1. (E-G) WT and Atf4 ${ }^{-/-}$BMMs were maximally differentiated for 9 days, followed by TRAP staining. TRAP+ MNCs (F) and the number of nuclei per OCL $(\mathbf{G})$ were scored. (H and $\mathbf{I}$ ) Bone resorption pit assay on dentin slices. BMMs were differentiated on dentin slices for 9 days. $(\mathbf{H})$ Bone resorption pits were stained with hematoxylin solution. (I) Pit area versus total bone area on each dentin slice was measured as described in Methods. (J) Time course of TRAP ${ }^{+}$mononuclear OCL differentiation. BMMs were differentiated for the indicated times followed by TRAP staining, and percent TRAP $^{+}$mononuclear cells was measured. ${ }^{*} P<0.01$ versus WT. Original magnification, $\times 100$ (C, E, and H), $\times 200$ (B and D).

Using biochemical, cellular, and genetic approaches, the present study demonstrates that ATF4 is an osteoclastic transcription factor, which we believe to be novel, that is essential for OCL differentiation. We showed that both in vitro and in vivo OCL differentiation was severely impaired by lack of ATF4 in a cell-autonomous manner and increased by OCL-targeted transgenic ATF4 expression. Our results revealed that, mechanistically, ATF4 functioned as a direct upstream activator of the gene encoding the critical transcription factor NFATc1. Further, ATF4 modulated RANKL activation of MAPK pathways, a key molecular event in OCL differentiation. Additionally, we demonstrated that ATF4 was critical for M-CSF induction of RANK expression, a key step to generate OCL progenitors, and the level of ATF4 protein was largely modulated by M-CSF and the PI3K/AKT path-

collagen, and proliferation and survival of OBLs $(39,40)$. Furthermore, our most recent work showed that ATF4 is critical for osteoblastic responses to parathyroid hormone (PTH) to increase bone formation (41). Elefteriou and coworkers showed that ATF4 mediates $\beta$-adrenergic induction of Rankl mRNA expression via direct binding to the upstream OSE1 site in the Rankl promoter in OBLs (42). Work from the same group further showed that OBL-targeted expression of ATF4 increased osteoblastic Rankl expression and thereby OCL differentiation (43). Although these studies clearly demonstrate that increased OBL expression of ATF4 enhances OCL differentiation via RANKL production in OBLs, to our knowledge, the possibility of an OCL-intrinsic direct role for ATF4 in regulating OCL differentiation has not previously been addressed. ways in BMMs. Therefore, these results indicate that ATF4 has important OCL-intrinsic functions both upstream and downstream of RANKL signaling during OCL differentiation.

\section{Results}

Inactivation of the Atf4 gene severely impairs OCL differentiation in vitro and in vivo in a cell-autonomous manner. We first tested whether ATF4, a previously known OBL-enriched transcription factor (39), is expressed in OCL-like cells. As shown in Supplemental Figure 1 (supplemental material available online with this article; doi:10.1172/JCI42106DS1), the levels of ATF4 protein in primary mouse BMMs and RAW264.7 cells (a mouse monocyte/macrophage cell line) were comparable to those in OBL-like cells (mouse 
Table 1

Bone histomorphometry in WT and Atf4--- tibiae

\begin{tabular}{lcl} 
& WT & Atf4 $^{-/}$ \\
Primary spongiosa & & \\
Oc.S/BS & $8.4 \pm 0.75$ & $4.2 \pm 0.48^{\mathrm{A}}$ \\
Oc.Nb/BPm & $9.2 \pm 0.96$ & $5.1 \pm 0.8^{\mathrm{A}}$ \\
Secondary spongiosa & & \\
Oc.S/BS & $8.5 \pm 0.56$ & $4.1 \pm 0.55^{\mathrm{A}}$ \\
Oc.Nb/BPm & $8.3 \pm 0.91$ & $4.3 \pm 0.74^{\mathrm{A}}$ \\
\hline
\end{tabular}

Oc.S/BS and Oc.Nb/BPm in primary and secondary spongiosa of tibiae in Figure $1 \mathrm{C}$ were measured as described in Methods. ${ }^{A} P<0.01$ versus WT.

MC-4 preosteoblastic cells, primary mouse bone marrow stromal cells [BMSCs], and rat UMR106-01 osteoblastic cells). As shown in Figure 1A, ATF4 protein was phosphorylated in primary mouse BMMs (note loss of upper bands with phosphatase treatment). Furthermore, a strong ATF4 signal was detected in the cytoplasm of the large multinuclear OCLs by immunohistochemical (IHC) staining using a specific ATF4 antibody (Figure 1B).

To determine whether ATF4 is required for osteoclastogenesis in vivo, the tibiae of 4-week-old WT and Atf4-/- mice were decalcified, and histological sections were stained for the OCL enzyme tartrate-resistant acid phosphatase (TRAP). We found that TRAP activity throughout the tibiae, including both the metaphyseal and the epiphyseal regions, was dramatically reduced in Atf4-/- compared with WT mice (Figure 1C). We next measured the effect of ATF4 deficiency on OCL differentiation in both primary and secondary spongiosa. OCL surface/bone surface (Oc.S/BS) and OCL number/bone perimeter (Oc.Nb/BPm) were reduced similarly in both primary and secondary spongiosa in Atf4-/- tibiae relative to WT tibiae (Figure 1D and Table 1). Given the dramatic decreases in Oc.S/BS and Oc.Nb/BPm in the Atf4-/bone, we next determined whether ATF4 is intrinsically required in BMMs for OCL differentiation by assessing whether OCL differentiation was normal upon addition of exogenous RANKL to Atf4-/- compared with WT BMM cultures in vitro by measuring the number of $\mathrm{TRAP}^{+}$multinucleated cells (MNCs; defined as having 3 or more nuclei per cell) generated by each. We found that TRAP ${ }^{+}$MNCs in BMM cul-

\section{Figure 2}

ATF4 deficiency impairs OCL differentiation in a cellautonomous manner. (A and B) CFU-GM assay. $2 \times 10^{4}$ BMMs from WT and Atf4-/- mice (6 per group) were cultured in methylcellulose semisolid medium in $35-\mathrm{mm}$ dishes in the presence of $1.0 \mathrm{ng} / \mathrm{ml}$ recombinant human GM-CSF for 10 days. The number of CFU-GM colonies was counted under an inverted microscope. Experiments were repeated 2 times. (C and D) OCL-OBL coculture. Primary calvarial OBLs from 3-day-old WT mice were cocultured with WT or Atf4-/- BMMs as described in Methods. ( $E$ and $\mathbf{F}$ ) Effects of increased RANKL. Primary BMMs (E) and purified CD11b+ BMMs (F) from both genotypes were differentiated in the presence of increasing concentrations of RANKL for 7 days, and the number of TRAP+ MNCs per well was counted. ${ }^{*} P<0.01$ versus WT. Original magnification, $\times 40(A) ; \times 200(C)$.
A
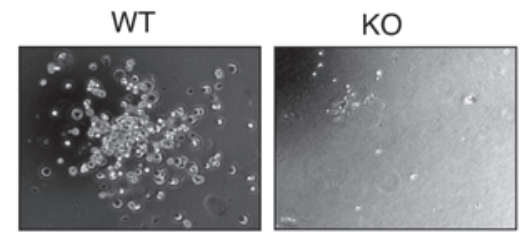

C

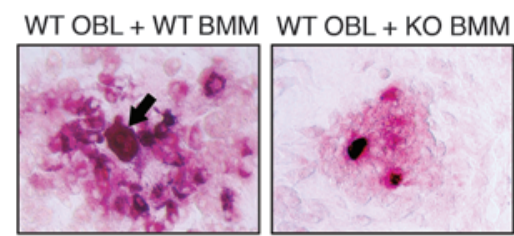

E

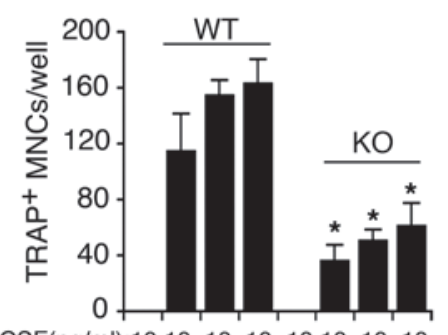

$\operatorname{CSF}(\mathrm{ng} / \mathrm{ml}): 1010 \quad 10 \quad 10 \quad 1010 \quad 10 \quad 10$

RANKL(ng/ml): 050100200050100200

BMMs
B

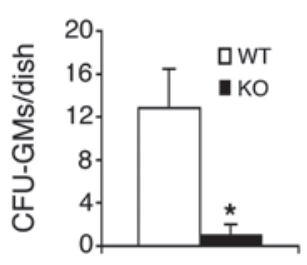

D

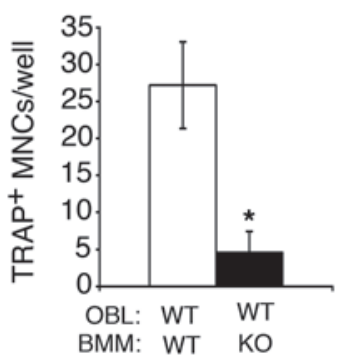

$\mathbf{F}$

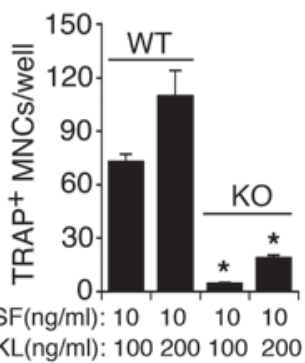


A

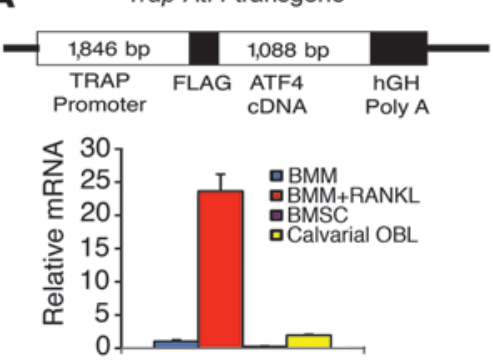

D
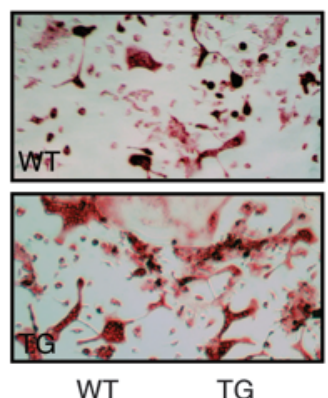

B

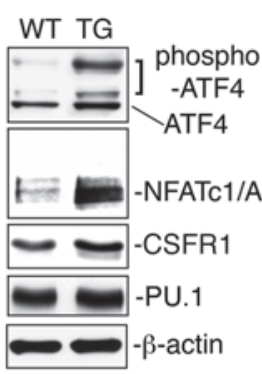

C

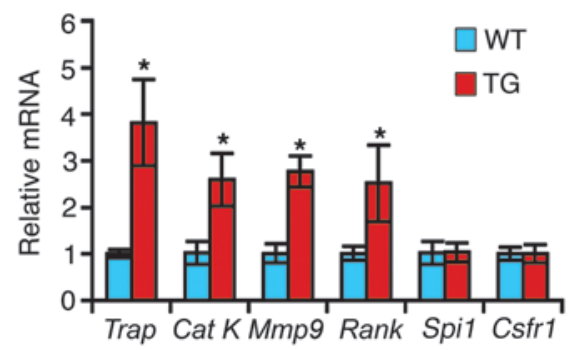

E

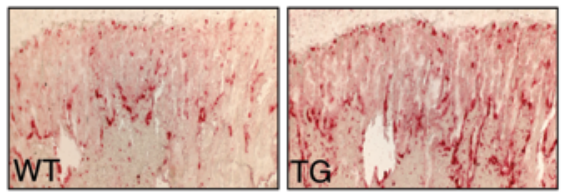

F

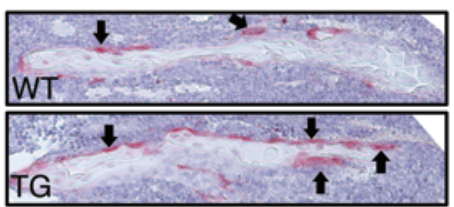

G WT

TG

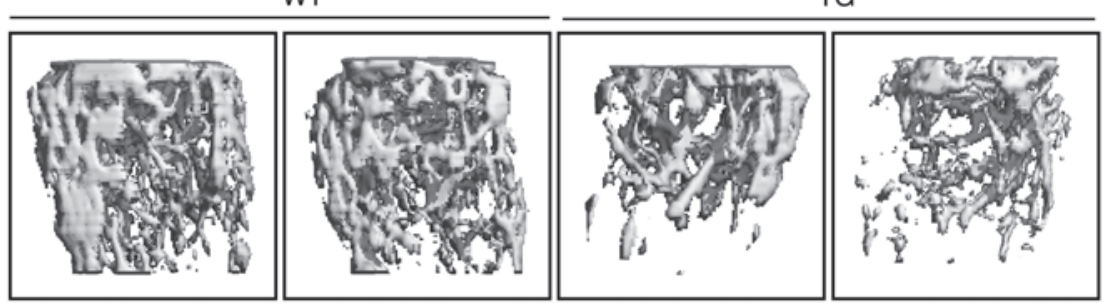

Figure 3

OCL-targeted transgenic overexpression of ATF4 dramatically increases OCL differentiation and bone resorption and results in a severe osteopenic phenotype. (A) Schematic representation of a transgene construct. An 1,846-bp fragment of the mouse Trap promoter was used to drive expression of full-length mouse ATF4 cDNA. Atf4 transgene expression in RANKL-differentiated and undifferentiated BMMs, BMSCs, or calvarial OBLs was measured by quantitative real-time RT-PCR using transgene-specific primers as described in Methods. (B-D) In vitro OCL differentiation. BMMs from 4-week-old WT and Trap-Atf4-tg mice (founder no. 2360) were differentiated into OCLs for 5 days followed by (B) Western blot analysis of ATF4, NFATc1, PU.1, CSFR1, and $\beta$-actin for loading; (C) real-time RT-PCR analysis for Trap, Cat K, Mmp9, Rank, Spi1, and Csfr1 mRNAs; and (D) TRAP staining of the BMM cultures. (E) TRAP staining. Tibial sections from 4-week-old WT and Trap-Atf4-tg mice were stained for TRAP activity. (F) TRAP ${ }^{+}$OCLs (arrows) on trabecular surfaces of WT and Trap-Atf4-tg tibiae. Oc.S/BS and Oc.Nb/BPm values for primary and secondary spongiosa are shown in Table 2. (G) $\mu \mathrm{CT}$ analysis. Fixed nondemineralized femurs from 3-month-old male WT and Trap-Atf4-tg mice were used for $\mu \mathrm{CT}$ analysis as previously described (41). BV/TV, Tb.N, and Tb.Sp values are shown in Table 3 . $n=3-7 .{ }^{*} P<0.01$ versus WT. Original magnification, $\times 100(\mathbf{D}$ and $\mathbf{E}) ; \times 200(\mathbf{F})$.

known to contain the earliest OCL precursors (44), and found that it was severely compromised in Atf4- $4^{--}$BMMs (Figure 2, A and B). We next examined whether coculture with WT OBLs rescues the OCL formation of Atf4 ${ }^{-/-}$BMMs (Figure 2, C and D). As expected, cocultures of WT OBLs with WT BMMs significantly induced the formation of TRAP ${ }^{+}$MNCs. Although WT OBLs did induce some

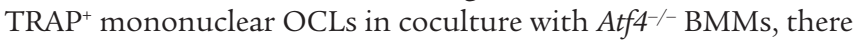
were almost no $\mathrm{TRAP}^{+} \mathrm{MNCs}$ observed. Furthermore, primary calvarial OBLs from 3-day-old Atf4-/- mice failed to induce $\mathrm{TRAP}^{+}$ MNC formation when cocultured with either WT or Atf4-/- BMMs (H. Cao and G. Xiao, unpublished observation), in support of the notion that osteoblastic ATF4 plays a role in OCL differentiation, probably via upregulation of RANKL expression (42). In addition, high concentrations of RANKL (up to $200 \mathrm{ng} / \mathrm{ml}$ ) did not restore the OCL differentiation defect in BMMs or purified CD $11 \mathrm{~b}^{+}$ BMMs of Atf4-/- mice (Figure 2, E and F). Collectively, these findings suggest that ATF4 deficiency impairs osteoclastogenesis in a cell-autonomous manner.

$O C L$-targeted overexpression of ATF4 dramatically increases OCL differentiation and bone resorption and causes a severe osteopenic phenotype. To further examine the OCL-intrinsic role of ATF4 in regulating osteoclastogenesis in vivo, we developed transgenic mice in which the Atf4 transgene is driven by an 1,846-bp mouse Trap promoter that selectively expresses ATF4 in OCLs (referred to herein as Trap-Atf4-tg mice; Figure 3A). This promoter has been successfully used to target OCL expression of many transgenes (45-47). Atf4 
Table 2

Bone histomorphometry in WT and Trap-Atf4-tg tibiae

\begin{tabular}{lcc}
\hline & WT & Trap-Atf4-tg \\
Primary spongiosa & & \\
Oc.S/BS & $9.1 \pm 0.36$ & $16 \pm 0.71^{\mathrm{A}}$ \\
Oc.Nb/BPm & $9.2 \pm 1.2$ & $16 \pm 0.94^{\mathrm{A}}$ \\
Secondary spongiosa & & \\
Oc.S/BS & $9.2 \pm 0.51$ & $16.5 \pm 0.72^{\mathrm{A}}$ \\
Oc.Nb/BPm & $9.3 \pm 1.03$ & $16.6 \pm 1.3^{\mathrm{A}}$ \\
\hline
\end{tabular}

Oc.S/BS and Oc.Nb/BPm in both primary and secondary spongiosa of tibiae in Figure $3 \mathrm{E}$ were measured as described in Methods. ${ }^{A} P<0.01$ versus WT.

transgene mRNA was highly expressed in RANKL-differentiated BMMs, but was minimal in undifferentiated BMMs, calvarial OBLs, or BMSCs (Figure 3A). As shown in Figure 3B, the level of ATF4 protein was dramatically increased in RANKL-differentiated BMM cultures from Trap-Atf4-tg mice compared with BMM from the control littermates. Transgenic ATF4 dramatically increased the protein levels of NFATc1/A, but not of PU.1 or CSFR1, in differentiated BMM cultures. The levels of OCL differentiation marker gene mRNAs (Trap, Rank, Cat $K$, and $M m p 9$ ) were all dramatically elevated in differentiated BMM cultures from Trap-Atf4-tg mice compared with those from WT mice. In contrast, like the proteins, the levels of Spi1 and Csfr1 mRNAs were not increased by transgenic ATF4 (Figure 3C). Using BMMs from 3 different transgenic lines, we found that OCL-targeted overexpression of ATF4 dramatically increased the number of TRAP ${ }^{+}$MNCs in vitro (WT, $56 \pm 11$ $\mathrm{TRAP}^{+}$MNCs/well; Trap-Atf4-tg, $270 \pm 17 \mathrm{TRAP}^{+}$MNCs/well; $P<0.01$; Figure 3D). We found a similar effect of transgenic ATF4 expression in vivo. TRAP activity was markedlyincreased in Trap-Atf4-tg compared with WT tibiae (Figure 3E). Oc.S/BS and Oc.Nb/BPm in both primary and secondary spongiosa of tibiae were dramatically increased in Trap-Atf4-tg versus WT mice $(P<0.01$; Figure 3F and Table 2). The serum level of C-telopeptide (CTX), an indicator of in vivo OCL activity, was elevated 2.4-fold in Trap-Atf4-tg mice relative to WT mice (WT, $19.5 \pm 3.7$; Trap-Atf4-tg, $46 \pm 3.3$; $P<0.01$ ). Quantitative $\mu \mathrm{CT}$ analysis of femur histomorphometric parameters showed that Trap-Atf4-tg mice had a significant reduction in bone volume/tissue volume (BV/TV) and trabecular number (Tb.N), as well as a marked increase in trabecular space (Tb.Sp), compared with WT littermates $(P<0.01$, all comparisons; Figure $3 \mathrm{G}$ and Table 3 ). Results from these experiments demonstrated that osteoclastic ATF4 overexpression increased OCL differentiation and bone resorption, resulting in a severe osteopenic phenotype.

ATF4 is an upstream activator of the Nfatc1 gene. We next examined whether deletion of Atf4 reduces expression of NFATc1, a master regulator of OCL differentiation. Results from quantitative realtime RT-PCR analysis showed that the level of $N$ fatc 1 mRNA relative to Gapdh mRNA was greatly decreased in Atf4-/- BMM cultures compared with WT cells (Figure 4A and Supplemental Figure 3). The expression of Cat $K$, a well-known NFATc1 downstream target gene, was almost abolished in Atf4-/- cells compared with WT control cells. Western blot analysis confirmed that NFATc1/A protein, the major isoform of NFATc1 expressed in OCLs, was drastically reduced by ATF4 deficiency (Figure 4B). In contrast, the levels of TRAF6 and c-Fos, both critical factors for OCL differentiation and NFATc1 induction $(7,8,48,49)$, were not decreased by ATF4 deficiency. Likewise, the mRNA and protein levels of PU.1 and CSFR1, both critical factors for early OCL lineage commitment and development, were not reduced by the lack of ATF4 (Figure 4, A and B), in accordance with their observed lack of increase by overexpression of ATF4 (Figure 3, B and C). As expected, the level of Atf4 mRNA was minimal in Atf4 $4^{-/-}$BMM cultures (Figure 4A). IHC staining of differentiated BMM cultures using a specific antibody for NFATc1 showed a strong signal in MNCs of WT cultures that was drastically reduced in Atf4 $4^{-/-}$cultures, although there were some mononuclear OCLs that were NFATc1 ${ }^{+}$in $A t f 4^{-/-}$cultures (Figure 4C). Similarly, in vivo, a strong NFATc1 signal was identified on all surfaces of trabeculae throughout WT tibiae, where OCLs are usually located (Figure 4D). In contrast, the majority of the trabecular surfaces of Atf4-/- tibiae were negative for NFATc1. Adenoviral ATF4 overexpression in BMMs increased the level of NFATc1 protein in a dose-dependent manner (Figure 4E). ATF4 activated -847/+66 Nfatc1 P1 promoter-pGL3-luciferase reporter activity in a dose-dependent manner, but failed to stimulate a $2.8-\mathrm{kb}$ mouse Runx2 promoter (Figure 4F). In contrast, the Nfatc1 P1 promoter was not activated by Runx2 (Supplemental Figure 4). As shown in Figure 4G, introduction of a 4-bp substitution mutation to the known AP1 binding site located at -644/-637 (from TGACTTCA to TGCGAACA) decreased ATF 4 activation by $50 \%$ without affecting basal promoter activity, which indicates that this site is critical for ATF4 regulation. ChIP assays showed that RANKL induced ATF4 interaction with a chromatin fragment of the proximal Nfatc1 promoter in RAW264.7 OCL-like cells (Figure $4 \mathrm{H})$. Consistent with results from previous studies $(33,38)$, both c-Fos and NFATc1 itself were also recruited to the same region of the Nfatc1 promoter in a RANKL-dependent manner. These data demonstrate that ATF4 is a critical upstream activator of the Nfatc1 gene and indicate that ATF4 not only regulates the number of OCL progenitors, but also has a direct role in activating genes downstream of RANK signaling.

We next examined whether NFATc1 can rescue the defective

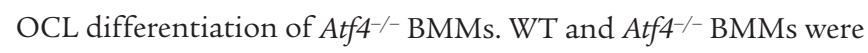
infected with increasing amounts of retrovirus expressing a constitutively active form of NFATc1 (50) and differentiated for 7 days, followed by TRAP staining. Although caNFATc1 dose-dependently increased the number of $\mathrm{TRAP}^{+}$MNCs in Atf4-/- BMM cultures (Figure 4I), surprisingly, at even the highest dose, it only slightly increased the number of nuclei per MNC (WT plus empty virus, $24 \pm 3.8$; WT plus NFATc1 virus, $32.6 \pm 5.5 ; P<0.01$; KO plus empty virus, $3.3 \pm 0.42$; KO plus NFATc1 virus, $4.8 \pm 0.71 ; P<0.01$ ).

ATF4 modulates RANKL activation of MAPKs, but not I $\mathrm{K}$ B pathways, in OCL progenitors. Because activation of the MAPK and IкB $\alpha / N F-\kappa B$ pathways by RANKL is crucial for NFATc1 expression and OCL differentiation, we next examined whether ATF4 deficiency affects

\section{Table 3}

Quantitative $\mu$ CT analysis of WT and Trap-ATF4-tg femur histomorphometric parameters

\begin{tabular}{lcc} 
& WT & Trap-ATF4-tg \\
BV/TV (\%) & $21 \pm 3.0$ & $12 \pm 3.8^{\mathrm{A}}$ \\
Tb.N (per mm) & $3.4 \pm 0.37$ & $2.3 \pm 0.07^{\mathrm{A}}$ \\
Tb.Sp (mm) & $0.30 \pm 0.03$ & $0.45 \pm 0.01^{\mathrm{A}}$ \\
\hline
\end{tabular}

Fixed nondemineralized femurs from 3-month-old male WT and TrapAtf4-tg animals were used for $\mu \mathrm{CT}$ analysis as previously described (41). 

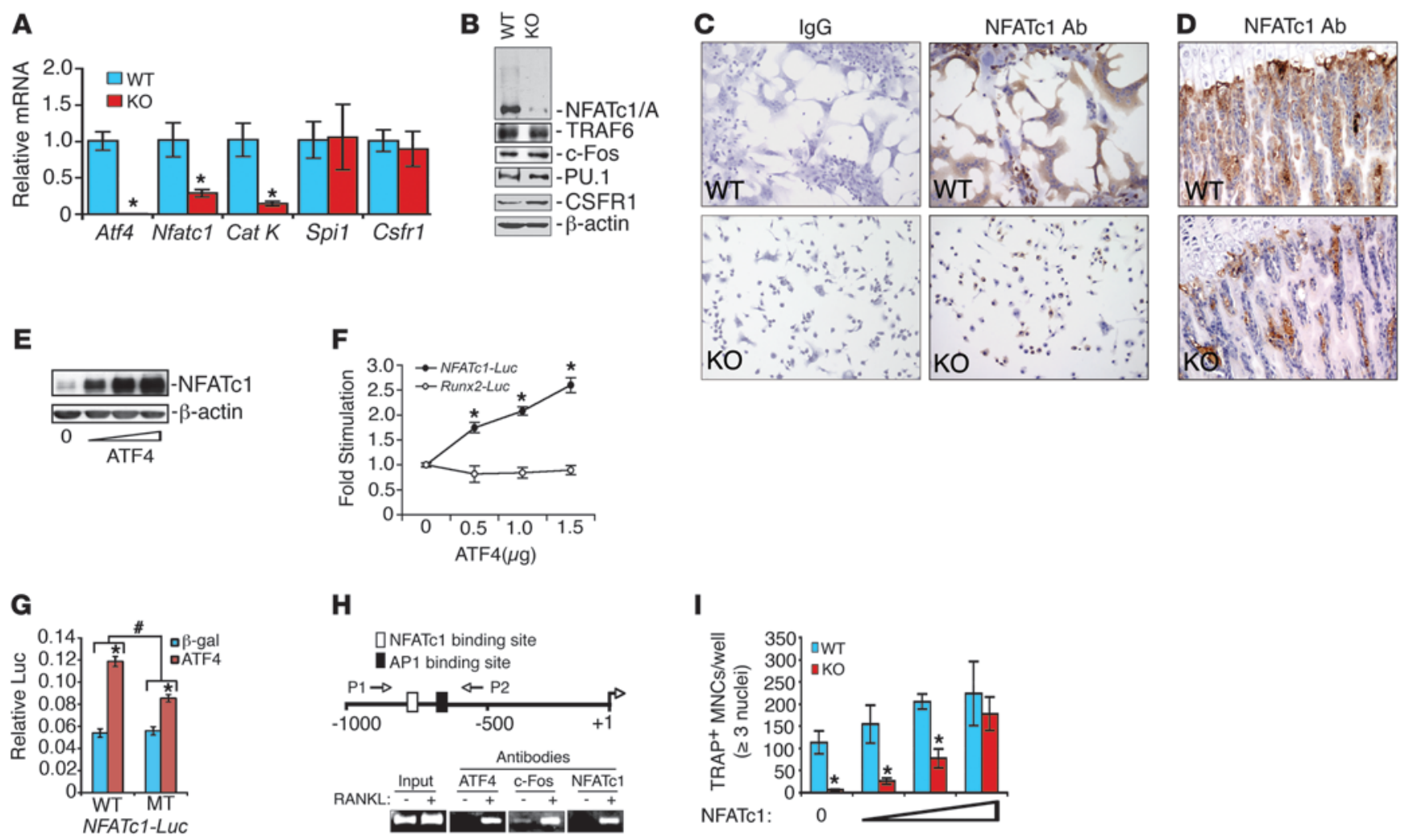

\section{Figure 4}

ATF4 regulates NFATc1 expression in BMM cultures and bones. (A and B) Total RNAs and protein lysates from differentiated WT and Atf4-/BMMs were used for real-time RT/PCR analysis (A) and Western blot (B). (C and D) Differentiated BMMs and tibial sections were subjected to IHC staining for NFATc1. (E) WT BMMs were infected with increasing amounts of ATF4 adenovirus, then switched to differentiation medium for 72 hours, followed by Western blot for NFATc1. (F) ATF4 activates the Nfatc1 P1 promoter. COS-7 cells were transfected with 0.8-kb Nfatc1-luc or 2.8-kb mouse Runx2-luc constructs and pRL-SV40 with the indicated amounts of ATF4 expression plasmid. After 30 hours, cells were harvested for the dual luciferase assay. ${ }^{*} P<0.01$ versus $0 \mu$ g ATF4. (G) COS-7 cells transfected with 0.8-kb Nfatc1-luc (WT) or the same plasmid containing a 4-bp substitution mutation (MT) in the putative ATF4-binding site and pRL-SV40 with or without ATF4 expression plasmid. ${ }^{*} P<0.05$ versus $\beta$-gal; $\# P<0.05$, WT versus MT ATF4/ $\beta$-gal. (H) ChIP assay. A schematic representation of the relevant region of the mouse Nfatc1 P1 promoter is shown. $\mathrm{P} 1$ and $\mathrm{P} 2$ indicate PCR primers used to analyze ChIP DNAs. RAW264.7 cells were treated with or without $50 \mathrm{ng} / \mathrm{ml}$ RANKL for 24 hours. ChIP assays were performed using antibodies against ATF4, c-Fos, or NFATc1. (I) WT and Atf4 ${ }^{-/-}$BMMs were cultured and infected with increasing amounts of retrovirus expressing caNFATc1, and switched to differentiation medium for 7 days. The number of TRAP+ MNCs per well was counted. ${ }^{*} P<0.01$ versus WT. Original magnification, $\times 100$.

RANKL activation of these important pathways in OCL progenitors. As shown in Figure 5, RANKL rapidly induced the phosphorylation of Erk $1 / 2$ in a time-dependent manner, which was delayed and reduced in Atf4-/- cells. ATF4 deficiency also slightly reduced the basal phosphorylation level of Erk1/2. Lack of ATF4 similarly compromised RANKL activation of p38 and JNK without markedly affecting their basal levels. In contrast, no difference was seen in RANKL-induced phosphorylation of IKB $\alpha$ in cells of the 2 genotypes. Collectively, lack of ATF 4 reduced the ability of RANKL to activate the Erk1/2, p38, and JNK MAPK pathways in OCL progenitors.

We next determined whether ATF4 deficiency affects M-CSFdependent signaling in BMMs. As shown in Supplemental Figure 5, M-CSF rapidly stimulated the phosphorylation of AKT and JNK in

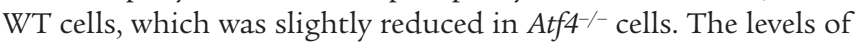
both phosphorylated and total Src were similar in WT and Atf4-/cells with or without M-CSF. In contrast to RANKL, M-CSF similarly activated Erk1/2 and p38 in WT and Atf4-/- cells. Thus, ATF4 deficiency did not dramatically impact M-CSF signaling in BMMs.
Levels of ATF4 protein in BMMs are modulated by M-CSF and PI3K/ $A K T$, and ATF4 is required for M-CSF induction of RANK expression. To determine whether ATF4 is regulated by M-CSF in early OCL differentiation, BMMs were cultured in the presence and absence of $30 \mathrm{ng} / \mathrm{ml} \mathrm{M-CSF}$ for $0,6,12,24$, and 48 hours, followed by Western blot for ATF4. The results showed that the level of ATF4 protein was dramatically reduced in the absence of M-CSF in a time-dependent manner. However, this reduction was completely prevented by M-CSF (Figure 6A). M-CSF did not alter the level of Atf4 mRNA (Figure 6B), which suggests that a posttranscriptional mechanism is involved in this regulation.

To define the signaling pathways through which M-CSF regulates ATF4, BMMs were treated with and without inhibitors or activators for various pathways in the presence of M-CSF for 24 hours. As shown in Figure 6C, LY294002, a specific inhibitor of the PI3K/AKT pathway, dramatically reduced total and phosphorylated ATF4. In contrast, the p38 inhibitor SB209580, the Erk1/2 inhibitor U0126, the PKA inhibitor H89, the PKC inhibitor GF109203X, and the PKA activator FSK did not markedly decrease or increase ATF4 or 
A
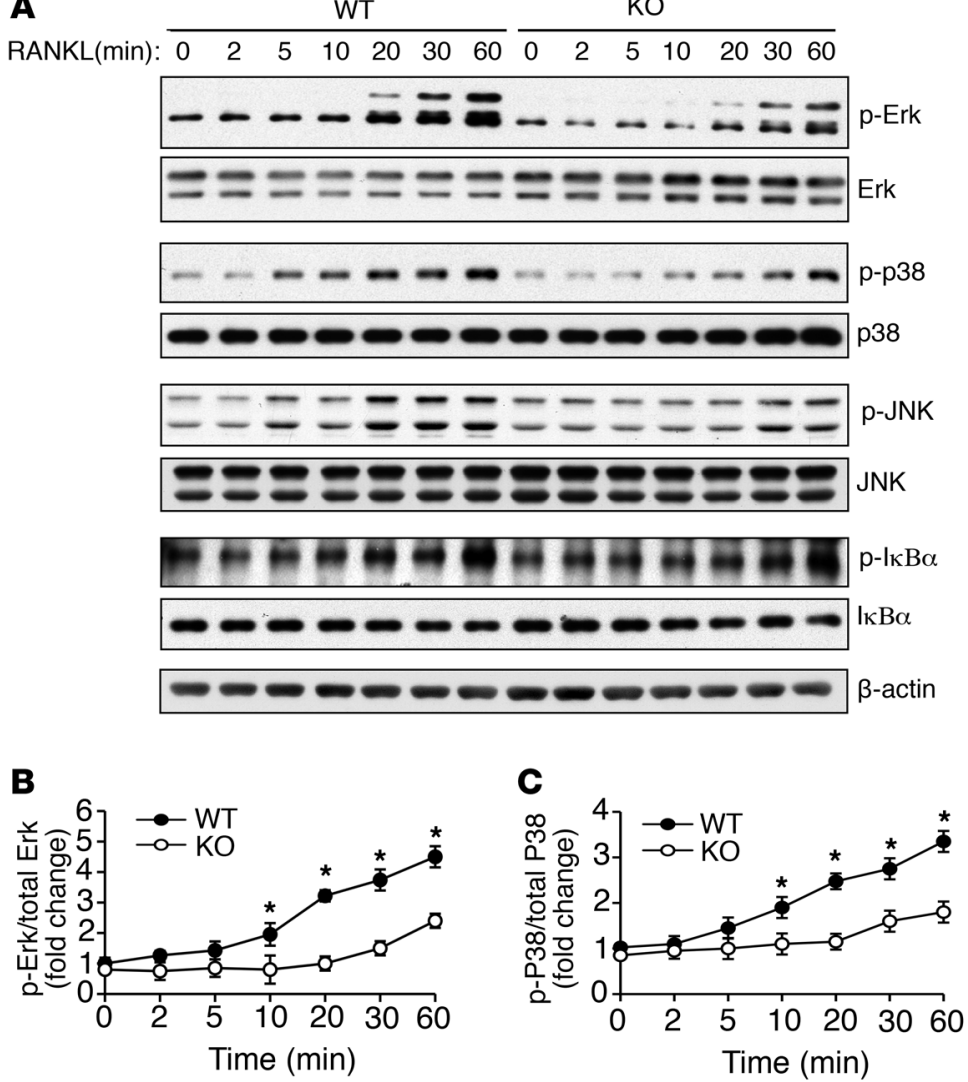

D

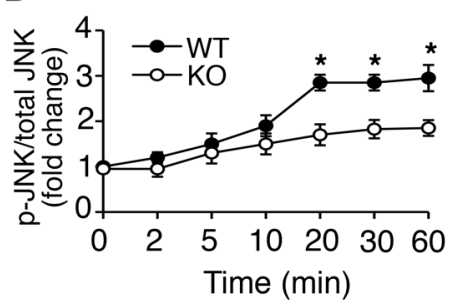

E

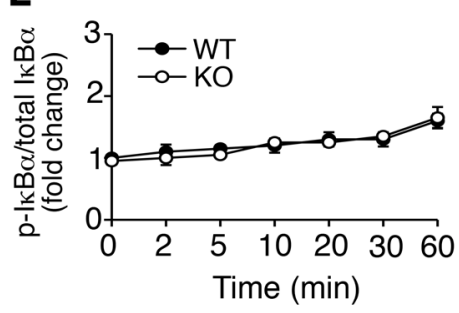

Figure 5

RANKL activation of the MAPK pathways is severely compromised in Att4-/- OCL progenitors. (A) Western blot. WT and Atf $^{-/-}$BMMs were cultured in proliferation medium for 3 days and switched to $2 \%$ FBS $\alpha$-MEM without M-CSF overnight, after which cells were exposed to $100 \mathrm{ng} / \mathrm{ml}$ RANKL for the indicated times. Cells were then lysed, fractionated by SDS-PAGE, and analyzed by Western blot analysis using antibodies recognizing phosphorylated and total ERK1/2, p38, JNK, and IкB $\alpha$. $\beta$-Actin served as the loading control. Similar results were obtained from 3 independent experiments. (B-E) Statistical analysis of the Western blots in $\mathbf{A} .{ }^{*} P<0.01$, WT versus $\mathrm{KO}$.

specific antibodies. M-CSF time-dependently induced Rank mRNA expression in WT BMM cultures (Figure 6I), consistent with results from a previous study (16). However, this induction was dramatically reduced in Atf4-/ cells. Taken together, these results suggest that ATF4 regulates early OCL differentiation at least in part by facilitating M-CSF induction of the Rank gene.

ATF4 deficiency results in increased $C D 11 b^{+}$cells in bone marrow and spleen and reduced $C D 3^{-} C D 45 R^{-} C D 11 b^{-10}$ $c$-kit ${ }^{+} \mathrm{CD} 115^{\text {hi }}$ cells in bone marrow. Because OCLs and macrophages share the same precursor, we next determined whether lack of ATF4 affects monocyte/macrophage precursors by measuring the $\mathrm{CD} 11 \mathrm{~b}^{+}$cell population in splenocytes and bone marrow from WT and Atf4-/ mice. In 5 independent experiments, the percentage of $\mathrm{CD}_{11 \mathrm{~b}^{+}}$cells was dramatically increased in $\mathrm{Atf4^{-/ }}$ splenocytes compared with WT cells, as measured by flow cytometry (WT, 3.04\% $\pm 0.64 \% ; \mathrm{KO}, 9.6 \% \pm 2.1 \% ; P<0.01$; Figure $7 \mathrm{~A})$. This increase in $\mathrm{CD} 11 \mathrm{~b}^{+}$cells was specific, because the percentages of both $\mathrm{T}$ lymphocytes $\left(\mathrm{CD}^{+}\right.$; Figure 7A) and dendritic cells (CD11 ${ }^{+}$; Supplemental Figure 6, A and B) were not increased by ATF4 deficiency (WT, $27.63 \% \pm 1.9 \% \mathrm{CD}^{+}, 1.59 \% \mathrm{CD}^{+} 1 \mathrm{c}^{+} ; \mathrm{KO}$, $\left.21.04 \% \pm 4.1 \% \mathrm{CD}^{+}, 1.52 \% \mathrm{CD}^{2} 1 \mathrm{c}^{+} ; P>0.05\right)$. Likewise, in bone marrow, the number of $\mathrm{CD} 11 \mathrm{~b}^{+}$cells was similarly increased in $\mathrm{Atf4^{-/ }}$ mice (WT, $41.5 \% \pm 0.08 \%$; KO, alter its phosphorylation. Importantly, exposure to LY294002 for only 24 hours prior to the addition of differentiation media inhibited in vitro OCL differentiation in a dose-dependent manner (Figure 6D). The concentrations of the inhibitors or activators used in this study are in the ranges previously reported to selectively affect the relevant pathways (51-54), or were as suggested by the respective manufacturers. We found no evidence of cell toxicity using these experimental conditions. Interestingly, the decrease in ATF4 in COS-7 cells induced by LY294002 was completely abolished by cycloheximide (CHX) and dramatically reduced by the proteasome inhibitor MG115 (Figure 6, E and F). These results suggest that regulation of ATF4 by LY294002 involves de novo protein biosynthesis as well as modulation of protein stability.

M-CSF-induced RANK expression is essential for generating OCL progenitors. We next evaluated whether ATF4 is required for this regulation. To this end, highly purified $\mathrm{CD} 11 \mathrm{~b}^{+} \mathrm{BMMs}$ were cultured in $\mathrm{M}-\mathrm{CSF}$-containing medium for 72 hours and stained with an anti-RANK antibody or control IgG. As shown in Figure 6, $\mathrm{G}$ and $\mathrm{H}$, the RANK signal was dramatically decreased in Atf4-/- relative to WT cells, as measured by both IHC and Western blot using
$63.6 \% \pm 3.4 \% ; P<0.01$ ), but the number of $\mathrm{CD}^{+}$cells (WT, $1.04 \% \pm 0.2 \%$; $\mathrm{KO}, 0.8 \% \pm 0.06 \% ; P>0.05$ ) and CD $11 \mathrm{c}^{+}$cells (Supplemental Figure $6 \mathrm{C}$ ) was not. At the same time, the percentage of CD11b-/lo cells, the osteoclastogenic population in the bone marrow, was dramatically reduced in Atf4-/- marrow and spleen. Since the $\mathrm{CD}^{-} \mathrm{CD} 45 \mathrm{R}^{-} \mathrm{CD} 11 \mathrm{~b}^{-/ / \mathrm{lo}} \mathrm{c}-\mathrm{kit}^{+} \mathrm{CD} 115^{\text {hi }}$ population (approximately $2 \%$ of fresh murine bone marrow preparations) contains the highest in vitro osteoclastogenic activity (55), we next determined whether Atf4 inactivation affects this OCL precursor population in bone marrow cells and splenocytes. In 5 independent experiments, the percentage of this cell population was slightly but significantly reduced in bone marrow cells by ATF4 deficiency (WT, $2.3 \% \pm 0.11 \%$; KO, $2.0 \% \pm 0.02 \% ; P=0.01$; Figure $7 \mathrm{~A}$ ). However, the percentage of this cell population in splenocytes was much lower than in bone marrow (approximately $0.25 \%$ ) and was not significantly changed by ATF4 deficiency. It should be noted that although $\mathrm{Atf4^{-/ }}$ bones are smaller and thinner than WT bones, total nucleated bone marrow cells per bone were not reduced in Atf4-/- compared with WT mice (Supplemental Figure 6D). ATF4 deficiency did not alter the proliferation and survival of CD11 b $\mathrm{b}^{+} \mathrm{BMMs}$ (Figure 7, B-E). Therefore, the lack of 
A

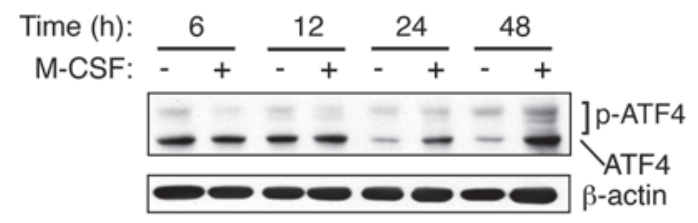

B

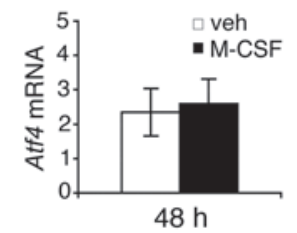

C

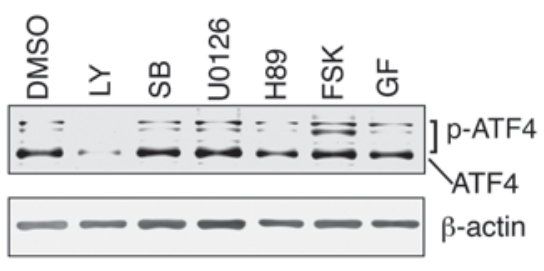

$\mathbf{F}$

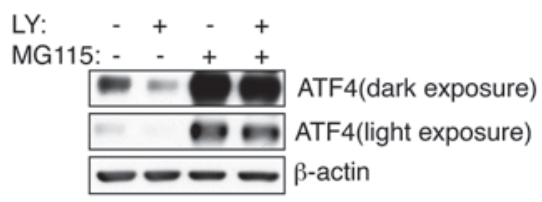

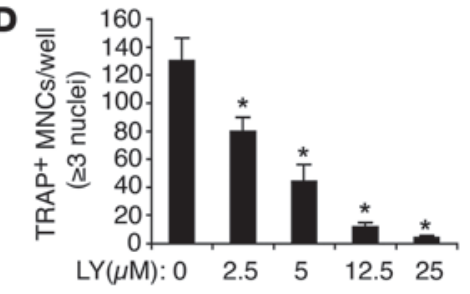

G

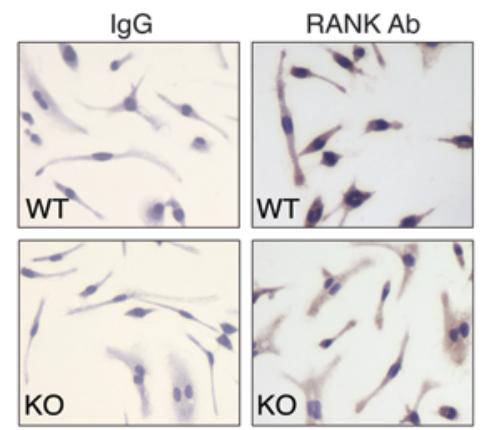

E

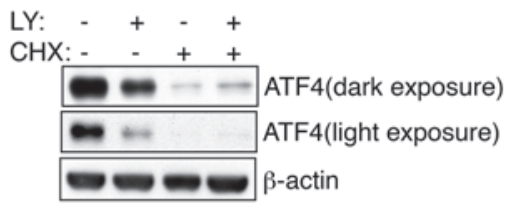

H

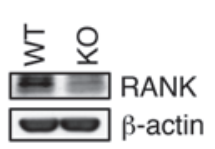

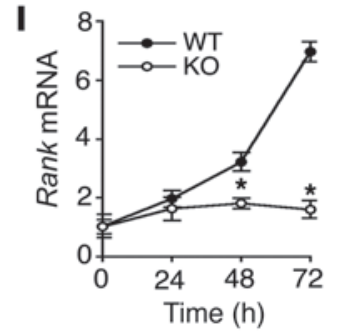

Figure 6

ATF4 is upregulated by M-CSF and PI3K/AKT and is required for M-CSF induction of RANK expression in BMMs. (A and B) Effects of M-CSF on ATF4 in BMMs. Cells were cultured with or without $30 \mathrm{ng} / \mathrm{ml} \mathrm{M-CSF}$ for the indicated times, followed by Western blot (A) or real-time RT-PCR (B) for ATF4. (C) Effects of various inhibitors or activators on the level of ATF4 in BMMs. Cells were cultured in M-CSF-containing medium with and without the indicated inhibitors or activators (10 $\mu \mathrm{M}$ ) for 24 hours. LY, LY294002; SB, SB209580; GF, GF109203X. (D) Effect of PI3K/AKT inhibition on OCL differentiation. BMMs were seeded in proliferation medium for 3 days and treated with increasing concentrations of LY294002 for 24 hours. Inhibitor was then removed by switching cells to differentiation medium for 5 days, followed by TRAP staining. ${ }^{*} P<0.01$ versus $0 \mu \mathrm{m}$. (E and F) COS-7 cells were transfected with $1.0 \mu \mathrm{g} \mathrm{pCMV/ATF4} \mathrm{expression} \mathrm{plasmid.} \mathrm{After} 24$ hours, cells were treated with or without $10 \mu \mathrm{M}$ LY294002 as well as with or without $10 \mu \mathrm{g} / \mathrm{ml}$ CHX (E) or $10 \mu \mathrm{M}$ MG115 (F) for another 24 hours. (G) IHC. Purified CD11 b+ BMMs were seeded in proliferation medium for 72 hours, followed by IHC with an anti-RANK antibody or control IgG. (H) Western blot. Primary BMMs were seeded in 35-mm dishes in proliferation medium for 72 hours. (I) Real-time RT-PCR. WT and Atf4-- BMMs were cultured in proliferation medium for 3 days and switched to $2 \%$ FBS $\alpha$-MEM without M-CSF overnight. Cells were then treated with $10 \mathrm{ng} / \mathrm{ml} \mathrm{M}$-CSF for the indicated times. ${ }^{*} P<0.01$, WT versus KO. Original magnification, $\times 200$.

ATF4 causes a lineage shift between OCLs and macrophages, resulting in an increase in macrophages. Interestingly, a similar increase in macrophages was observed in mice lacking c-Fos, whose deficiency also reduces NFATc1 and OCL differentiation (7-9).

\section{Discussion}

The results of our present study establish, for the first time to our knowledge, that ATF4, a transcription factor previously shown to be important in OBLs, also plays a direct and critical role in regulating OCL differentiation both in vitro and in bones. Drastically reduced OCL differentiation in BMM cultures from Atf4-/- animals was not rescued by coculture with WT OBLs or high concentrations of RANKL, which suggests that ATF4 plays an intrinsic role in OCLs that is indispensable for RANKL-induced OCL differentiation. Dramatic reduction in the formation of CFU-GMs in Atf4 $4^{-/}$BMM cultures suggests that ATF4 deficiency impairs the formation of OCL precursors. To examine the in vivo actions of ATF4 in OCLs, this study used the mouse Trap promoter to drive expression of ATF4 in OCLs. Using this approach, ATF4 was shown to stimulate expression of NFATc1 and other OCL-specific genes and OCL differentiation in BMM cultures. Of particular significance, Trap-Atf4-tg animals displayed a striking in vivo effect on OCL differentiation and bone resorption, resulting in a severe osteopenic phenotype. Because the Trap promoter is not active in OBLs, we were able to discriminate between the effects of ATF4 in OCLs and those in OBLs or BMSCs (i.e., via ATF4-dependent production of RANKL). Furthermore, as shown in Figure 1C and Figure 3E, TRAP activity in hypertrophic chondrocytes close to primary spongiosa was very weak, which suggests that the Trap promoter is not active in these cells. Therefore, Atf4 transgene expression driven by this promoter should be low in the hypertrophic chondrocytes; consequently, the potential contribution of the Atf4 transgene expression in these cells to the observed bone phenotype in Trap-Atf4-tg mice (i.e., osteopenia, increased OCL differentiation, and bone resorption) should be minimal. 
A
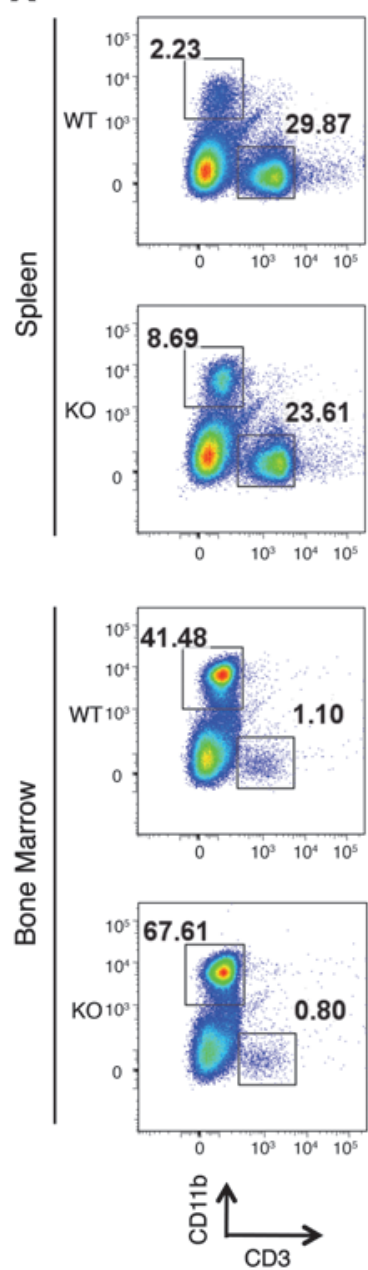

B

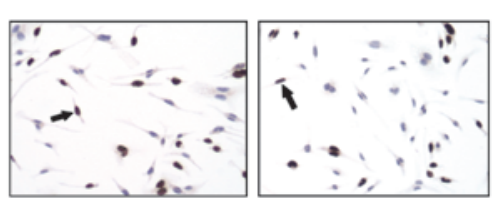

D
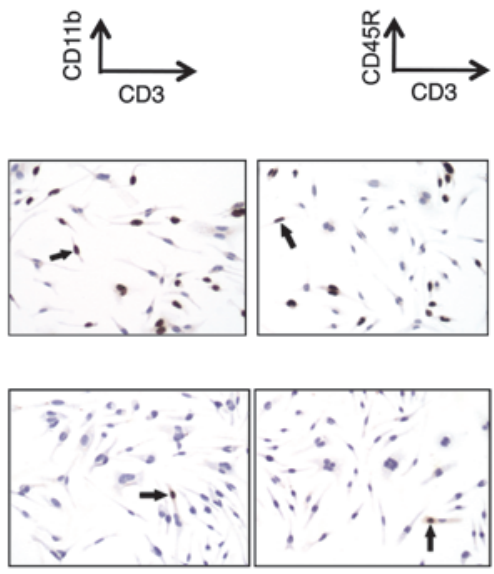
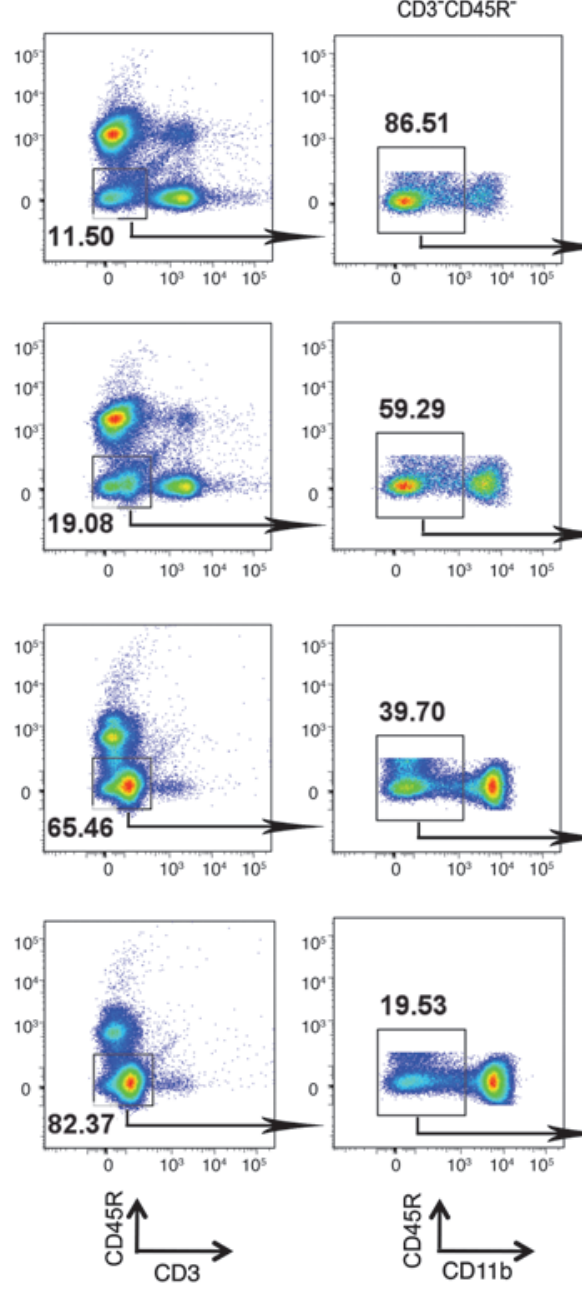

$\mathrm{CD}^{-}{ }^{-} \mathrm{CD} 45 \mathrm{R}^{-}$
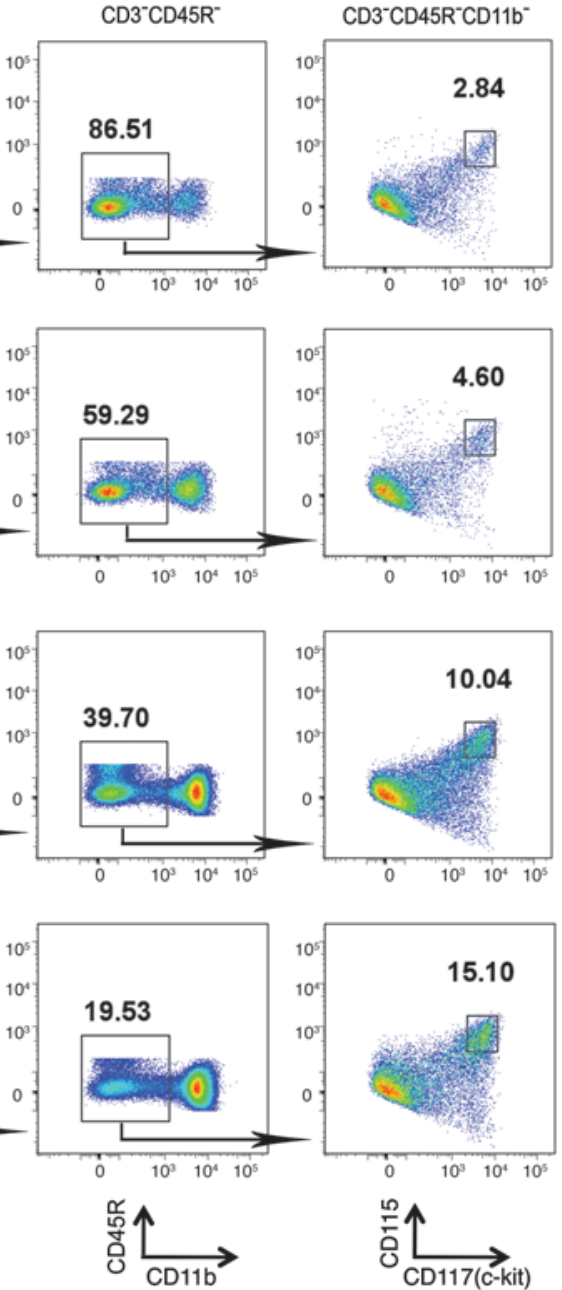

C

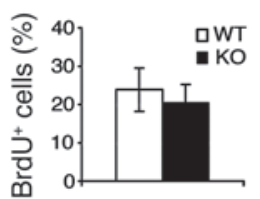

E

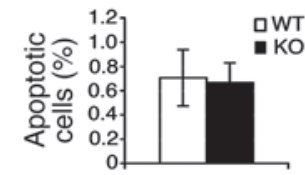

Figure 7

ATF4 deficiency increases CD11b+ cells in bone marrow and spleen and reduces $\mathrm{CD}^{-} \mathrm{CD} 45 \mathrm{R}^{-} \mathrm{CD} 11 \mathrm{~b}^{-1 / 10}$ C-kit ${ }^{+}$CD115 ${ }^{\text {hi }}$ cells in bone marrow. (A) Flow cytometry. Splenocytes and bone marrow cells from WT and Atf4-/ mice were stained with bio-strepPB-conjugated CD11b, FITC-conjugated CD3, PE-conjugated CD45R, Pecy5-conjugated c-kit (CD117), and APC-conjugated CD115 antibodies and analyzed with flow cytometry as described in Methods. The percentage of CD3-CD45R-CD11 ${ }^{-/ 10}$ c-kit+CD115i cells in bone marrow cells or splenocytes was calculated by multiplying the percentages of gated populations as indicated. A representative experiment is shown; values in Results were averaged over 5 independent experiments. $1 \mathrm{WT}$ and $1 \mathrm{Atf}^{-/-}$mouse were used in each experiment. (B and C) BrdU staining. Purified CD11b+ BMMs were cultured in 8-well chambers $\left(5 \times 10^{5}\right.$ cells/well) in proliferation medium for 72 hours, followed by BrdU staining as described previously $(40,41)$. Arrows indicate BrdU+ (i.e., proliferating) cells. (D and $\mathbf{E}$ ) TUNEL staining. CD11b+ BMMs were treated as in B, followed by TUNEL staining as described previously (40, 41). Arrows indicate apoptotic cells. Original magnification, $\times 100$.
One striking result in the present study, which we believe to be novel, is the finding that ATF4 is a direct upstream activator of the Nfatc1 gene, a master regulator of OCL differentiation. Levels of NFATc1 were drastically reduced in Atf4-/- OCLs and bones. Conversely, OCL-specific expression of ATF4 in transgenic mice greatly increased the expression of NFATc1 and its downstream target genes as well as OCL differentiation. ATF4 activated Nfatc1 gene transcription via interaction with the P1 promoter. Interestingly, a previously known AP1 binding site located at -644/-637 was critical for ATF4 activation of the Nfatc1 P1 promoter, which suggests that ATF4 directly binds to this site and/or functions via interactions with AP1 factors. Future study will differentiate among these possibilities. Of particular significance, ATF4 interaction with the P1 promoter was stimulated by RANKL. In addition to its direct regulation, ATF4 may also indirectly increase NFATc1 expression by promoting RANKL-RANK signaling. This notion is supported by our findings that (a) M-CSF induction of RANK, the receptor for RANKL in OCL precursors, was severely impaired in Atf4 $4^{--}$BMMs; and (b) RANKL activation of the Erk1/2, JNK, and p38 MAPK pathways in OCL progenitors, which is crucial for the subsequent expression of NFATc1, was greatly compromised by the lack of ATF4. Although ATF4 is essential for its expression, 
retroviral transduction of NFATc1 1 DNA into BMM only partially rescued the OCL differentiation defect in $\mathrm{Atf4^{-/ }}$ BMM cultures, as large OCLs were still not formed. Possible explanations include: (a) the magnitude and duration of NFATc1 expression was inappropriate for full rescue; and (b) other OCL differentiation-related genes and/or factors are also regulated by ATF4.

Our results established that ATF4 is a critical downstream target of M-CSF-PI3K/AKT signaling in early OCL differentiation. The level of ATF4 protein was largely dependent upon the presence of M-CSF and the PI3K/AKT pathway in BMMs. M-CSF upregulated ATF4 at least in part by stabilizing its protein, which involves the PI3K/AKT pathway. Strikingly, short-term pharmacologic inhibition of the PI3K/AKT pathway dramatically reduced the level of ATF4 protein in undifferentiated BMMs and subsequent OCL differentiation. These results strongly suggest that the $\mathrm{M}-\mathrm{CSF}-\mathrm{PI} 3 \mathrm{~K} / \mathrm{AKT}-\mathrm{ATF} 4$ axis identified in the present study plays a crucial role in regulating early OCL differentiation. PI3K/AKT signaling has been implicated in OCL activation and bone resorption in neurofibromatosis type I (NF1), a congenital disorder resulting from loss of function of the tumor suppressor gene NF1, which encodes neurofibromin, a GTPase-activating protein for Ras. NF1 patients have a significantly higher incidence of osteoporosis and osteopenia (21-23). Recent studies showed that increased OCL activity and osteoporosis is caused by increased AKT signaling in OCLs in murine and human NF1 haploinsufficiency $(18,19)$. It would be interesting to test whether ATF4 plays a role in the AKT-induced OCL differentiation and bone resorption in NF1 patients. Notably, mice selectively lacking NF1 in OBLs displayed increased OCL activity, probably via upregulation of ATF4 in OBLs, and thereby exhibited increased RANKL expression (43).

Results from the present study demonstrated that ATF4 is not required for early OCL lineage commitment and development. First, CSFR1 and PU.1 expression, which is required for the generation of the common progenitors for both macrophages and OCLs, was not altered in Atf4-/- BMMs or bones. Second, M-CSF activation of AKT and MAPKs was not markedly different in WT and $\mathrm{Atf}^{-{ }^{--}}$BMMs. Third, both proliferation and survival of highly purified CD $11 \mathrm{~b}^{+}$BMMs of the 2 genotypes were similar. Finally,

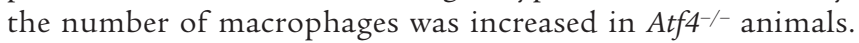
Therefore, ATF4 deficiency impairs OCL, but not macrophage, differentiation, and the OCL differentiation defect in $\mathrm{Atf4^{-/ }}$ mice occurs later than in Spi1- or Csfr1-deficient mice.

Although ATF4 is crucial for OCL differentiation, Atf4-/- mice did not display an osteopetrotic phenotype, which is often observed in mice and humans in which genes encoding key OCL-regulating factors - such as CSF1R, RANK, RANKL, TRAF6, Src, PU.1, NF- $\mathrm{B}$ (p50/p52 double knockout), and c-Fos - are inactivated or mutated (1-9). Lack of osteopetrosis in Atf4 $4^{-/-}$mice could be explained, at least in part, by the fact that ATF4 is also important for OBL function and bone formation $(39,40,56)$. Therefore, it is expected that $\mathrm{Atf4^{-/- }}$ mice have a low-bone turnover osteoporosis.

ATF4 can be specifically regulated by different signal transduction factors in OBLs and OCLs. For examples, ATF4 is directly phosphorylated and activated by RSK2 in OBLs, which is critical for ATF4 activity as well as bone formation (56). Furthermore, PTH - via its receptor, PTH1R, which is expressed in OBLs - upregulates Atf4 gene expression and activity, which is required for this hormone to induce osteocalcin gene expression as well as bone formation $(41,53)$. PKA phosphorylation of ATF4 at its Ser254 residue mediates $\beta$-adrenergic induction of Rankl mRNA expression in OBLs (42). Additionally, ATF4 increases OBL function and bone formation through interactions with the OBL-specific transcription factor Runx2 (57-60). In contrast, in OCLs, ATF4 is regulated by M-CSF and the PI3K/AKT pathway, mediates M-CSF induction of RANK, and facilitates RANKL induction of MAPKs and expression of NFATc1. In contrast to the effects of M-CSF and PI3K/AKT on ATF4 protein levels in BMMs and OCLs, both RSK2 and PKA do not affect the ATF4 protein level in OBLs $(42,56)$. Furthermore, ATF4 $4^{\mathrm{Ser} 219}$ and ATF4 ${ }^{\mathrm{Ser} 224}$ phosphorylation is involved in binding to $\beta \operatorname{TrCP}$ ubiquitin ligase, thus increasing proteolytic degradation in HeLa cells $(61,62)$, whereas the activation of AKT in BMMs enhances ATF4 stability. This suggests that ATF4 is modulated by differential phosphorylations in OBLs and OCLs and/or that M-CSF$\mathrm{PI} 3 \mathrm{~K} / \mathrm{AKT}$ signaling regulates a protein that modulates ATF4 protein stability in BMMs/OCLs. Therefore, it should be possible to design therapeutic agents that selectively inhibit ATF4 in OCLs.

The results of the present study establish a direct and important role for ATF4 in regulating multiple steps in OCL differentiation and suggest that manipulation of ATF4 in OCLs may be a strategy for increasing bone mass in diseased states.

\section{Methods}

Reagents. Tissue culture media and FBS were obtained from Thermo Scientific HyClone. LY294002, SB209580, U0126, H89, FSK, GF109203X, and DMSO were purchased from Sigma-Aldrich. Calf intestinal phosphatase was purchased from Promega. All other chemicals were of analytical grade. Atf4-deficient and Trap-Atf4-tg mice. Breeding pairs of Atf4-heterozygous mice (Swiss black), as described previously $(40,53)$, were used to generate WT Atf $4^{+/+}$, heterozygous $\mathrm{Atf4^{+/- }}$, and homozygous mutant $\mathrm{Atf4^{-/- }}$ mice for this study. 4- to 8-week-old mice were sacrificed for BMMs. Mice selectively expressing ATF4 in OCLs were developed at the Transgenic \& Chimeric Mouse Facility of the University of Pittsburgh using an 1,846-bp mouse Trap promoter, provided by S. Reddy (Medical University of South Carolina, Charleston, South Carolina, USA; ref. 63). We identified 17 positive animals by PCR of tail DNA using a $5^{\prime}$ primer in the Atf4 cDNA sequence (5'-ATGACCCACCTGGAGTTAGTTTGACA-3') and a $3^{\prime}$ primer in the hGH-PoyA sequence (5'-GCACTGGGGAGGGGTCACA-3'). Expression of Atf4 transgene was measured by Western blot analysis using ATF 4 antibody. 5 founders (animal no. 2330, 2333, 2358, 2359, and 2360) were found to express Atf4 transgene at different levels. These transgenic animals were in C57BL/ 6 background. All research protocols were approved by the Institutional Animal Care and Use Committee of the VA Pittsburgh Healthcare System, where this study was conducted.

In vitro OCL assays and serum CTX assay. Nonadherent BMMs were isolated from total bone marrow cells cultured on tissue culture dishes for 48 hours. $\mathrm{CD}_{11 \mathrm{~b}^{+}}$or $\mathrm{CD} 11 \mathrm{c}^{+}$cells were isolated from $\mathrm{BMMs}$ using the $\mathrm{CD} 11 \mathrm{~b}$ or $\mathrm{CD} 11 \mathrm{c}$ microbeads and the LS columns according to the manufacturer's instructions (Miltenyi Biotec Inc). For differentiation, cells were first cultured in proliferation medium ( $\alpha$-MEM containing 10\% FBS and $10 \mathrm{ng} / \mathrm{ml} \mathrm{human} \mathrm{recombi-}$ nant M-CSF) for 3 days, then switched to differentiation medium (proliferation medium plus $50 \mathrm{ng} / \mathrm{ml}$ human recombinant RANKL) for 4-9 days. The $\mathrm{TRAP}^{+} \mathrm{MNCs}$ were scored using an inverted microscope. For Pit assay, BMMs $\left(5 \times 10^{5}\right.$ cells/well $)$ were seeded on dentin slices in 24 -well plates in proliferation medium for 3 days and switched to differentiation medium for 9 days. Bone resorption pits were stained with hematoxylin solution. Pit area versus total bone area and pit area versus $\mathrm{TRAP}^{+} \mathrm{MNC}$ area of each dentin slice were measured using Image Pro Plus 6.2 software (Media Cybernetics Inc). Coculture experiments were performed as previously described (64). Briefly, primary calvarial OBLs $\left(3.2 \times 10^{4}\right.$ cells/well $)$ were seeded in 24 -well plates and cultured in $\alpha$-MEM containing $10 \%$ FBS and $50 \mu \mathrm{g} / \mathrm{ml}$ ascorbic acid. BMMs $\left(4 \times 10^{5}\right.$ cells/well $)$ were seeded on top of the OBLs. The medium was 
supplemented with $10 \mathrm{ng} / \mathrm{ml} \mathrm{M-CSF}$ and $10^{-8} \mathrm{M}$ 1,25 dihydroxyvitamin $\mathrm{D}_{3}$. OCLs were identified by TRAP staining and counted. Serum levels of CTX, degradation products from type I collagen during osteoclastic bone resorption, were measured using the RatLaps EIA Kit according to the manufacturer's instruction (Immunodiagnostic Systems Limited).

Histological evaluation, bone histomorphometry, and IHC. WT and Atf4-/- mice were euthanized, and tibiae were fixed in $10 \%$ formalin at $4{ }^{\circ} \mathrm{C}$ for 24 hours, decalcified in 10\% EDTA ( $\mathrm{pH} 7.4$ ) for 10-14 days, and embedded in paraffin. Sections of tibiae from WT and Atf $4^{-/-}$mice were used for TRAP staining as described previously (65). Bone histomorphometry, such as Oc.S/BS and $\mathrm{Oc} \cdot \mathrm{Nb} / \mathrm{BPm}$, in both primary and secondary spongiosa of tibiae was measured using Image Pro Plus 6.2 software (Media Cybernetics Inc.) as previously described $(43,66)$. Cells cultured in 8-well culture chambers (Nalgene Nunc), or $5-\mu \mathrm{m}$ sections of tibiae, were subjected to IHC staining with antibodies against ATF4, NFATc1, or RANK (Santa Cruz Biotechnology) using the EnVision ${ }^{+}$System-HRP (DAB) kit (Dako North America Inc.) as described previously (41). Fixed nondemineralized femurs were used for $\mu \mathrm{CT}$ analysis at the Center for Bone Biology using VIVACT40 (SCANCO Medical AG), as previously described (41).

Cell proliferation and TUNEL staining. BrdU staining was used to measure cell proliferation as previously described $(40,41)$. Cell survival was evaluated using the ApopTag Peroxidase In Situ Apoptosis Detection Kit according to the manufacturer's instruction (Millipore).

Quantitative RT-PCR. RNA isolation and RT were previously described (53). Quantitative real-time RT-PCR analysis was performed to measure relative mRNA levels using SYBR Green kit (Bio-Rad Laboratories Inc.). Samples were normalized to Gapdh expression. The DNA sequences of mouse primers used for real-time PCR are summarized in Supplemental Table 1.

Western blot analysis. Western blot analysis was performed as previously described $(53,58)$. Antibodies used were as follows: antibodies against NFATc1, c-Fos, TRAF6, RANK, and CSFR1 and anti-rabbit or anti-mouse antibodies conjugated with horseradish peroxidase from Santa Cruz Biotechnology Inc.; antibodies recognizing phosphorylated and total ERK1/2, p38 MAPK, JNK, AKT, Src, IKB $\alpha$, and PU.1 from Cell Signaling Technology Inc.; and mouse monoclonal antibody against $\beta$-actin from Sigma-Aldrich.

DNA constructs, transfection, adenovirus, and retrovirus. $\mathrm{pCMV} / \beta$-gal, $\mathrm{pCMV/}$ ATF4, and pCMV/Runx2 were described previously $(41,57,58,67)$. NFATc1-luc, containing a mouse $N f a t c 1$ promoter element $(-847 /+66)$ driving a firefly luciferase reporter gene, was constructed in the project laboratory by PCR subcloning promoter fragment using mouse tail DNA as a template into pGL3-luc vector (Promega). Mutant Nfatc1-luc, which contains a 4-bp substitution mutation in the AP1 binding site located at -644/-637 (from TGACTTCA to TGCGAACA), was generated from WT Nfatc1-luc (-847/+66) by PCR amplification using a QuickChange XL Site-Directed Mutagenesis Kit (Stratagene) with the following primers: forward, 5'-GGGAAGCCTGCGATTTTACATAATGCGAACAGCATGCAAGGCGTCTCGGCACCC-3'; reverse, 5'-GGGTGCCGAGACGCCTTGCATGCTGTTCGCATTATGTAAAATCGCAGGCTTCCC-3'. Sequence accuracy was confirmed by automatic DNA sequencing. Runx2-luc was provided by P. Ducy (Columbia University, New York, New York, USA; ref. 68). For all transfection experiments, the amount of plasmid DNAs ( $0.125 \mu \mathrm{g}$ reporter plasmid, $1.0 \mathrm{ng}$ normalization plasmid PRL-SV4, and 0.5-1.5 $\mu$ g expression plasmid) was balanced as necessary with $\beta$-gal expression plasmid such that the total DNA was constant in each group. Experiments were performed in triplicate and repeated 3-4 times. Adenoviruses expressing ATF 4 and $\beta$-gal were described previously (57). Retrovirus expressing caNFATc1 and its control empty retrovirus were described previously (38). The amount of adenovirus or retrovirus was balanced as necessary with a control adenovirus expressing $\beta$-gal or an empty retrovirus such that the total amount was constant in each group.

ChIP. ChIP assays were performed as described previously $(41,58)$. The equivalent of $10 \mu \mathrm{g}$ DNA was used as starting material (input) in each ChIP reaction with $2 \mu \mathrm{g}$ of the appropriate antibody (ATF4, c-Fos, or NFATc1). Fractions of the purified ChIP DNA (5\%) or inputs ( $0.02 \%-0.05 \%)$ were used for PCR analysis. The reaction was performed with AmpliTaq Gold DNA Polymerase (Applied Biosystems) for 35 cycles of 60 seconds at $95^{\circ} \mathrm{C}$, $90 \mathrm{sec}$ onds at $58^{\circ} \mathrm{C}$, and 120 seconds at $68^{\circ} \mathrm{C}$. A PCR primer pair (P1, CCGGGACGCCCATGCAATCTGTTAGTAATT; P2, GCGGGTGCCCTGAGAAAGCTACTCТСССТT) was generated to detect DNA segments located near the AP-1-binding site at -644/-637 and 2 NFATc1-binding sites at -689/-684 (TTTTCC) and -680/-676 (TTTTCC), respectively, in mouse Nfatc1 proximal promoter (33). The PCR products were separated on 3\% agarose gels and visualized with ultraviolet light. All ChIP assays were repeated at least 3 times.

Flow cytometry. Cell surface fluorescence was determined using FITCconjugated CD3, bio-strep-PB- or APC-conjugated CD11b, Pecy5-conjugated c-kit (i.e., CD117), PE-conjugated CD45R, APC-conjugated CD115, and FITC-conjugated CD11c with appropriate isotype controls (eBioscience Inc.). For flow cytometry samples, erythrocytes were first removed by lysis. BMMs or splenocytes from WT and Atf $4^{-/-}$mice were then stained with the appropriate antibody. Between 5,000 and 10,000 events were collected from samples using a BD FACScan (BD Biosciences), and analysis was performed using Cell Quest (BD Biosciences).

Statistics. Data were analyzed with GraphPad Prism software, version 4.0. A 1-way ANOVA analysis was used, followed by the Tukey test. Results are expressed as mean $\pm \mathrm{SD}$. Differences with a $P$ value less than 0.05 were considered statistically significant.

\section{Acknowledgments}

We thank Renny T. Franceschi (University of Michigan, Ann Arbor, Michigan, USA) for critical reading of this manuscript. Thanks to Harry Blair (University of Pittsburgh, Pittsburgh, Pennsylvania, USA) for expert advice on bone histomorphometry. This work was in part supported by NIH grant DK072230, Department of Defense grant W81XWH-07-1-0160, and Chinese Ministry of Science and Technology grant 2009CB918902 (to G. Xiao) as well as by NIH grant AR057310 (to D.L. Galson).

Received for publication December 18, 2009, and accepted in revised form May 26, 2010.

Address correspondence to: Guozhi Xiao, Rm 2E-107, VA Pittsburgh Healthcare System, 151-U, Pittsburgh, Pennsylvania 15240, USA. Phone: 412.360.3036; Fax: 412.360.6960; E-mail: xiaog@upmc.edu.
1. Roodman GD. Regulation of osteoclast differentiation. Ann N Y Acad Sci. 2006;1068:100-109.

2. Yavropoulou MP, Yovos JG. Osteoclastogenesis Current knowledge and future perspectives. J Musculoskelet Neuronal Interact. 2008;8(3):204-216.

3. Asagiri M, Takayanagi $\mathrm{H}$. The molecular understanding of osteoclast differentiation. Bone. 2007; 40(2):251-264.

4. Kobayashi T, et al. TRAF6 is a critical factor for dendritic cell maturation and development. Immu- nity. 2003;19(3):353-363.

5. Kong YY, et al. OPGL is a key regulator of osteoclastogenesis, lymphocyte development and lymph-node organogenesis. Nature. 1999;397(6717):315-323.

6. Kim N, Odgren PR, Kim DK, Marks SC Jr, Choi Y. Diverse roles of the tumor necrosis factor family member TRANCE in skeletal physiology revealed by TRANCE deficiency and partial rescue by a lymphocyte-expressed TRANCE transgene. Proc Natl Acad Sci U S A. 2000;97(20):10905-10910.
7. Wang ZQ, Ovitt C, Grigoriadis AE, Mohle-Steinlein U, Ruther U, Wagner EF. Bone and haematopoietic defects in mice lacking c-fos. Nature. 1992;360(6406):741-745.

8. Johnson RS, Spiegelman BM, Papaioannou V. Pleiotropic effects of a null mutation in the c-fos proto-oncogene. Cell. 1992;71(4):577-586.

9. Grigoriadis AE, et al. c-Fos: a key regulator of osteoclast-macrophage lineage determination and bone remodeling. Science. 1994;266(5184):443-448. 
10. Lee SH, et al. v-ATPase V0 subunit d2-deficient mice exhibit impaired osteoclast fusion and increased bone formation. Nat Med. 2006;12(12):1403-1409.

11. Ash P, Loutit JF, Townsend KM. Osteoclasts derived from haematopoietic stem cells. Nature. 1980;283(5748):669-670.

12. Lacey DL, et al. Osteoprotegerin ligand is a cytokine that regulates osteoclast differentiation and activation. Cell. 1998;93(2):165-176.

13. Yasuda $\mathrm{H}$, et al. Osteoclast differentiation factor is a ligand for osteoprotegerin/osteoclastogenesis-inhibitory factor and is identical to TRANCE/RANKL. Proc Natl Acad Sci U S A. 1998;95(7):3597-3602.

14. Simonet WS, et al. Osteoprotegerin: a novel secreted protein involved in the regulation of bone density. Cell. 1997;89(2):309-319.

15. Usui M, et al. Murine and chicken chondrocytes regulate osteoclastogenesis by producing RANKL in response to BMP2. J Bone Miner Res. 2008; 23(3):314-325.

16. Arai F, et al. Commitment and differentiation of osteoclast precursor cells by the sequential expression of c-Fms and receptor activator of nuclear factor kappaB (RANK) receptors. J Exp Med. 1999; 190(12):1741-1754.

17. Chang M, Hamilton JA, Scholz GM, Masendycz P, Macaulay SL, Elsegood CL. Phosphatidylinostitol-3 kinase and phospholipase C enhance CSF-1dependent macrophage survival by controlling glucose uptake. Cell Signal. 2009;21(9):1361-1369.

18. Yan J, et al. Rac1 mediates the osteoclast gains-infunction induced by haploinsufficiency of Nf1. Hum Mol Genet. 2008;17(7):936-948.

19. Yang FC, et al. Hyperactivation of $\mathrm{p} 21$ ras and PI $3 \mathrm{~K}$ cooperate to alter murine and human neurofibromatosis type 1-haploinsufficient osteoclast functions. J Clin Invest. 2006;116(11):2880-2891.

20. Takeshita S, et al. SHIP-deficient mice are severely osteoporotic due to increased numbers of hyperresorptive osteoclasts. Nat Med. 2002;8(9):943-949.

21. Kuorilehto T, Poyhonen M, Bloigu R, Heikkinen J, Vaananen K, Peltonen J. Decreased bone mineral density and content in neurofibromatosis type 1 : lowest local values are located in the load-carrying parts of the body. Osteoporos Int. 2005;16(8):928-936

22. Lammert $M$, et al. Decreased bone mineral density in patients with neurofibromatosis 1 . Osteoporos Int. 2005;16(9):1161-1166

23. Illes $T$, Halmai $V$, de Jonge $T$, Dubousset $J$. Decreased bone mineral density in neurofibromatosis-1 patients with spinal deformities. Osteoporos Int. 2001;12(10):823-827.

24. Kawamura N, et al. Akt1 in osteoblasts and osteoclasts controls bone remodeling. PLOS ONE. 2007;2(10):e1058.

25. Boyle WJ, Simonet WS, Lacey DL. Osteoclast differentiation and activation. Nature. 2003; 423(6937):337-342.

26. Hogan PG, Chen L, Nardone J, Rao A. Transcriptional regulation by calcium, calcineurin, and NFAT. Genes Dev. 2003;17(18):2205-2232.

27. Crabtree GR. Generic signals and specific outcomes: signaling through $\mathrm{Ca} 2+$, calcineurin, and NF-AT. Cell. 1999;96(5):611-614.

28. Crabtree GR, Olson EN. NFAT signaling: choreographing the social lives of cells. Cell. 2002;109 suppl:S67-S79.

29. Ishida $\mathrm{N}$, et al. Large scale gene expression analysis of osteoclastogenesis in vitro and elucidation of NFAT2 as a key regulator. J Biol Chem. 2002; 277(43):41147-41156.

30. Shinohara M, Takayanagi H. Novel osteoclast signaling mechanisms. Curr Osteoporos Rep. 2007; 5(2):67-72.

31. de la Pompa JL, et al. Role of the NF-ATc transcription factor in morphogenesis of cardiac valves and septum. Nature. 1998;392(6672):182-186.

32. Ranger AM, et al. The transcription factor NF-ATc is essential for cardiac valve formation. Nature. 1998;392(6672):186-190.

33. Asagiri $M$, et al. Autoamplification of NFATc1 expression determines its essential role in bone homeostasis. J Exp Med. 2005;202(9):1261-1269.

34. Ikeda F, Nishimura R, Matsubara T, Hata K, Reddy SV, Yoneda T. Activation of NFAT signal in vivo leads to osteopenia associated with increased osteoclastogenesis and bone-resorbing activity. J Immunol. 2006;177(4):2384-2390.

35. Takayanagi $\mathrm{H}$, et al. Induction and activation of the transcription factor NFATc1 (NFAT2) integrate RANKL signaling in terminal differentiation of osteoclasts. Dev Cell. 2002;3(6):889-901.

36. Hirotani H, Tuohy NA, Woo JT, Stern PH, Clipstone NA. The calcineurin/nuclear factor of activated $\mathrm{T}$ cells signaling pathway regulates osteoclastogenesis in RAW264.7 cells. J Biol Chem. 2004; 279(14):13984-13992.

37. Yamashita T, et al. NF-kappaB p50 and p52 regulate receptor activator of NF-kappaB ligand (RANKL) and tumor necrosis factor-induced osteoclast precursor differentiation by activating c-Fos and NFATc1. J Biol Chem. 2007;282(25):18245-18253.

38. Matsuo K, et al. Nuclear factor of activated T-cells (NFAT) rescues osteoclastogenesis in precursors lacking c-Fos. J Biol Chem. 2004;279(25):26475-26480.

39. Yang X, Karsenty G. ATF4, the osteoblast accumulation of which is determined post-translationally, can induce osteoblast-specific gene expression in non-osteoblastic cells. J Biol Chem. 2004, 279(45):47109-47114.

40. Zhang $X$, et al. Activating transcription factor 4 is critical for proliferation and survival in primary bone marrow stromal cells and calvarial osteoblasts. J Cell Biochem. 2008;105(3):885-895.

41. Yu S, et al. Critical role of activating transcription factor 4 in the anabolic actions of parathyroid hormone in bone. PLoS One. 2009;4(10):e7583.

42. Elefteriou F, et al. Leptin regulation of bone resorption by the sympathetic nervous system and CART. Nature. 2005;434(7032):514-520.

43. Elefteriou F, et al. ATF4 mediation of NF1 functions in osteoblast reveals a nutritional basis for congenital skeletal dysplasiae. Cell Metab. 2006 4(6):441-451

44. Menaa C, Kurihara N, Roodman GD. CFU-GMderived cells form osteoclasts at a very high efficiency. Biochem Biophys Res Commun. 2000;267(3):943-946.

45. Ikeda F, et al. Critical roles of c-Jun signaling in regulation of NFAT family and RANKL-regulated osteoclast differentiation. J Clin Invest. 2004; 114(4):475-484.

46. Hentunen TA, et al. Immortalization of osteoclast precursors by targeting $\mathrm{Bcl}-\mathrm{XL}$ and Simian virus 40 large $\mathrm{T}$ antigen to the osteoclast lineage in transgenic mice. J Clin Invest. 1998;102(1):88-97.

47. Kurihara N, et al. Mutation of the sequestosome 1 (p62) gene increases osteoclastogenesis but does not induce Paget disease. J Clin Invest. 2007; 117(1):133-142.

48. Wong BR, et al. TRANCE, a TNF family member, activates Akt/PKB through a signaling complex involving TRAF6 and c-Src. Mol Cell. 1999; 4(6):1041-1049.

49. Lomaga MA, et al. TRAF6 deficiency results in osteopetrosis and defective interleukin-1, CD40, and LPS signaling. Genes Dev. 1999;13(8):1015-1024.

50. Porter CM, Clipstone NA. Sustained NFAT signaling promotes a Th1-like pattern of gene expression in primary murine CD4+ T cells. J Immunol. 2002; 168(10):4936-4945

51. Jiang D, Franceschi RT, Boules H, Xiao G. Parathyroid hormone induction of the osteocalcin gene: requirement for an osteoblast-specific element 1 sequence in the promoter and involvement of multiple signaling pathways. J Biol Chem. 2004;279(7):5329-5337.

52. Xiao G, Jiang D, Gopalakrishnan R, Franceschi RT. Fibroblast growth factor 2 induction of the osteocalcin gene requires MAPK activity and phosphorylation of the osteoblast transcription factor, Cbfa1/Runx2. J Biol Chem. 2002; 277(39):36181-36187.

53. Yu S, et al. Parathyroid hormone increases activating transcription factor 4 expression and activity in osteoblasts: requirement for osteocalcin gene expression. Endocrinology. 2008;149(4):1960-1968.

54. Selvamurugan N, Pulumati MR, Tyson DR, Partridge NC. Parathyroid hormone regulation of the rat collagenase- 3 promoter by protein kinase A-dependent transactivation of core binding factor alpha1. J Biol Chem. 2000;275(7):5037-5042.

55. Jacquin C, Gran DE, Lee SK, Lorenzo JA, Aguila HL. Identification of multiple osteoclast precursor populations in murine bone marrow. J Bone Miner Res. 2006;21(1):67-77.

56. Yang X, et al. ATF4 Is a Substrate of RSK2 and an Essential Regulator of Osteoblast Biology; Implication for Coffin-Lowry Syndrome. Cell. 2004; 117(3):387-398.

57. Xiao G, et al. Cooperative Interactions between Activating Transcription Factor 4 and Runx2/Cbfa1 Stimulate Osteoblast-specific Osteocalcin Gene Expression. J Biol Chem. 2005;280(35):30689-30696.

58. Yu S, et al. General transcription factor IIA-gamma increases osteoblast-specific osteocalcin gene expression via activating transcription factor 4 and runt-related transcription factor 2.J Biol Chem. 2008; 283(9):5542-5553.

59. Dobreva G, et al. SATB2 is a multifunctional determinant of craniofacial patterning and osteoblast differentiation. Cell. 2006;125(5):971-986.

60. Tominaga $\mathrm{H}$, et al. CCAAT/enhancer-binding protein $\{$ beta\} promotes osteoblast differentiation by enhancing Runx2 activity with ATF4. Mol Biol Cell. 2008;19(12):5373-5386

61. Lassot I, et al. ATF4 degradation relies on a phosphorylation-dependent interaction with the SCF(betaTrCP) ubiquitin ligase. Mol Cell Biol. 2001; 21(6):2192-2202.

62. Pons J, Evrard-Todeschi N, Bertho G, Gharbi-Benarous J, Benarous R, Girault JP. Phosphorylationdependent structure of ATF4 peptides derived from a human ATF4 protein, a member of the family of transcription factors. Peptides. 2007;28(12):2253-2267.

63. Reddy SV, Kuzhandaivelu N, Acosta LG, Roodman GD. Characterization of the 5 -flanking region of the human tartrate-resistant acid phosphatase (TRAP) gene. Bone. 1995;16(5):587-593.

64. Mak KK, et al. Hedgehog signaling in mature osteoblasts regulates bone formation and resorption by controlling PTHrP and RANKL expression. Dev Cell. 2008;14(5):674-688.

65. Liu B, Yu SF, Li TJ. Multinucleated giant cells in various forms of giant cell containing lesions of the jaws express features of osteoclasts. J Oral Pathol Med. 2003;32(6):367-375.

66. Parfitt AM, et al. Bone histomorphometry: standardization of nomenclature, symbols, and units. Report of the ASBMR Histomorphometry Nomenclature Committee. J Bone Miner Res. 1987;2(6):595-610.

67. Singha UK, et al. Rapamycin inhibits osteoblast proliferation and differentiation in MC3T3-E1 cells and primary mouse bone marrow stromal cells. J Cell Biochem. 2008;103(2):434-446.

68. Zambotti A, Makhluf H, Shen J, Ducy P. Characterization of an osteoblast-specific enhancer element in the CBFA1 gene. J Biol Chem. 2002; 277(44):41497-41506. 